\title{
Beam Selection Assisted UAV-BS Deployment and Trajectory for Beamspace mmWave Systems
}

\author{
Xingxuan Zuo, ${ }^{1}$ Jiankang Zhang $\mathbb{D}^{2},{ }^{2}$ Sheng Chen, ${ }^{3}$ and Xiaomin $\mathrm{Mu}^{1}$ \\ ${ }^{1}$ School of Information Engineering, Zhengzhou University, Zhengzhou 450001, China \\ ${ }^{2}$ Department of Computing \& Informatics, Bournemouth University, BH12 5BB, UK \\ ${ }^{3}$ School of Electronics and Computer Science, University of Southampton, SO17 1BJ Southampton, UK \\ Correspondence should be addressed to Jiankang Zhang; jzhang3@bournemouth.ac.uk
}

Received 8 August 2021; Accepted 7 September 2021; Published 30 September 2021

Academic Editor: Xingwang Li

Copyright @ 2021 Xingxuan Zuo et al. This is an open access article distributed under the Creative Commons Attribution License, which permits unrestricted use, distribution, and reproduction in any medium, provided the original work is properly cited.

Exploiting unmanned aerial vehicles (UAVs) as base stations (UAV-BS) can enhance capacity, coverage, and energy efficiency of wireless communication networks. To fully realize this potential, millimeter wave (mmWave) technology can be exploited with UAV-BS to form mmWave UAV-BS. The major difficulty of mmWave UAV-BS, however, lies in the limited energy of UAVBS and the multiuser interference (MUI). Beam division multiple access with orthogonal beams can be employed to alleviate the MUI. Since each user has dominant beams around the line of sight direction, beam selection can reduce the power consumption of radio frequency chain. In this paper, we formulate the problem of maximizing the sum rate of all users by optimizing the beam selection for beamspace and UAV-BS deployment in the mmWave UAV-BS system. This nonconvex problem is solved in two steps. First, we propose a signal-to-interference plus noise ratio-based greedy beam selection scheme to ensure that all the ground users in the given area can be served by the UAV-BS, where a zero forcing precoding scheme is used to eliminate the MUI. Then, we utilize the continuous genetic algorithm to find the optimal UAV-BS deployment and beam pattern to maximize the sum rate of all users. Moreover, considering the mobility of the UAV-BS, the UAV-BS trajectory and beam selection for beamspace are optimized in the mmWave UAV-BS system. The simulation results demonstrate the effectiveness of the proposed design for the mmWave UAV-BS system.

\section{Introduction}

Unmanned aerial vehicles (UAVs) have drawn growing attention in a wide range of applications, such as disaster rescue, surveillance and monitoring, aerial imaging, and cargo delivery $[1,2]$. Wireless communication utilizing UAVs is a promising technology to achieve fast deployment and flexible reconfiguration $[3,4]$. Specifically, UAVs as different types of wireless communication platforms, such as UAV base stations (UAV-BS), aerial relays, and UAV swarms, assist and enhance terrestrial communications [5, 6]. Employing UAV-BS in particular is a cost-effective solution to assist the existing terrestrial communication infrastructure by providing seamless coverage and improving the network performance. Recent researches on UAV-BS can be divided into two main categories.
In the first category, UAVs are considered aerial quasistationary BSs. The altitude and horizontal positions of UAVs can be separately or jointly optimized to obtain a better air-ground channel for meeting different requirements of ground users [7-9]. Specifically, in [7], the altitude of the UAV-BS was optimized to achieve the maximum coverage for the terrestrial users. By contrast, with the fixed altitude, the horizontal placement of UAV-BS was jointly optimized with the user association in [8]. Furthermore, the joint optimization of altitude and horizontal positions of UAV-BS was investigated in [9], where the aim of the UAV-BS placement is to maximize the number of covered users with minimum transmit power.

For the second category, UAVs are considered mobile BSs. By exploiting the mobility of UAV-BS, the communication 
distance between the UAV-BS and terrestrial users can be significantly shortened via careful design on the trajectory of UAV-BS. In [10], a joint optimization of the UAV-BS trajectory and transmit power for multiple users was developed to dynamically establish short-distance line of sight (LoS) links and thus to improve the throughput of the system. A joint optimization of the UAV-BS trajectory and user scheduling for a UAV-enabled secure system was proposed in [11] to maximize the minimum secrecy rate under the required constraints of UAV-BS and ground users. In [12], a joint optimization of the UAV trajectory and nonorthogonal multiple access (NOMA) precoding was investigated for a UAVassisted NOMA network, where the UAV trajectory and NOMA precoding are jointly optimized to maximize the sum rate of users served by the UAV-BS and NOMA-network BS.

Early researches mainly focused on the UAV-BS working in microwave frequencies with a single antenna owing to the strict constraint of size. Because the UAV-BS also has limited on-board energy, the signals of a single antenna may be significantly attenuated due to the long communication distance between the UAV-BS and ground users [13]. However, by exploiting the small wavelengths of millimeter wave (mmWave) signals, mmWave frequencies can be adopted to the UAV-BS to pack large antenna arrays in small dimensions $[14,15]$.

The combination of UAV-BS and mmWave communication technology therefore offers advantages of enhancing coverage, improving energy efficiency, and providing sufficient bandwidth [16]. An UAV-BS mmWave system can naturally establish LoS links between UAV-BS and ground users to support the connectivity of existing ground wireless networks [17]. Because of limited scattering of air-to-ground links in the mmWave band, the path loss is dominant by LoS. The authors of [18] developed a 3D beamforming approach to achieve efficient and flexible coverage in mmWave UAV-BS communications. In [19], the impact of adaptive navigation on mmWave UAV-BSs was investigated to enhance the system performance. Furthermore, the mmWave UAV-BS deployment optimization is an important issue to improve the performance. In [20], the optimized mmWave UAV-BS deployment, which includes the optimal height, horizontal coordinates, and coverage radius, was analyzed by taking into account human body blockage. Xiao et al. [21] presented a mmWave UAV-BS deployment optimization with a constant modulus beamforming to maximize the sum rate of all ground users. However, since mmWave UAV-BSs place large antenna arrays in a small area, the power consumption of radio frequency (RF) chains is considerable [22], but UAV-BSs typically have limited energy supply. In addition, to support simultaneous transmissions between the mmWave UAV-BS and ground users, the multiuser interference (MUI) mitigation is necessary.

Since mmWave UAV-BS signals propagate mainly through LoS paths, users from different directions can be simultaneously served by orthogonal beams, which is known as beam division multiple access (BDMA) [16]. By employing orthogonal beams, MUI can be effectively decreased to improve the system performance [23]. In particular, the discrete lens array (DLA), which points signals in different directions, was employed in BDMA to transform the conventional spatial channel into a beamspace channel [24]. To exploit the sparsity of the beamspace channel, beam selection was investigated in [25] to achieve nearoptimal performance with fewer RF chains. In [26], a comparison among different kinds of beam selection schemes was evaluated, in terms of the required number of RF chains as well as the trade-off between spectral efficiency and energy efficiency. Furthermore, the power allocation for BDMA transmission was analyzed in [27] with only statistical channel state information (CSI). In [28], considering the power leakage and the imperfect channel statistics estimation, the RF chains were further reduced after the beamspace transformation in the lens antenna array.

There exist increasing interests in the use of the genetic algorithm (GA) for wireless communication and signal processing [29-31], since GA can attain the global optimal solutions of challenging optimization problems with affordable computational complexity and it does not require derivative information [32]. The work [31] applied several evolutionary algorithms, including continuous GA (CGA), to solve the difficult problem of joint channel estimation and turbo multiuser detection/decoding. Compared with discrete GA [32], CGA has high precision, low storage, and high speed without the requirement of prior decoding. In [33], GA was utilized to find the optimal deployment of drones to cover the target area with the lowest cost. However, the method of [33] is based on the single transmit antenna, which can be improved by taking the multiple transmit antennas and MUI into account.

The comparison of the recent researches in UAV-BS and BDMA is summarized in Table 1 . Inspired by the existing research, we focus on the mmWave UAV-BS system, where the mmWave-frequency antenna array and DLA are employed by the UAV-BS to direct signals to different directions and to form the beamspace channel. We propose a beam selection optimization for beamspace and UAV-BS deployment to maximize the sum rate of all ground users. Due to the sparse nature of the beamspace channel in BDMA, the dominant beams are near the LoS directions of the ground users. By properly designing the horizontal positions of the mmWave UAV-BS, not only can the better channel gain be achieved but also the interfering channel distance among the ground users can be enlarged to alleviate the MUI, while imposing lower number of RF chains. However, this design problem is nonconvex and difficult to solve directly. To tackle this challenging optimization problem, we decompose it into two subproblems by first designing the beam selection scheme for beamspace and then finding the optimal positions and beam pattern of the mmWave UAV-BS. For the first subproblem, we propose a signal-to-interference plus noise ratio- (SINR-) based greedy beam selection scheme. When the UAV-BS communicates with its served users, if the served users have different dominant beams, the UAV-BS will naturally select their corresponding dominant beams. However, in the scenario that two or more users have the same dominant beam, the UAV-BS can select the beams to users according to the SINR-based greedy beam selection scheme. Zero forcing (ZF) precoding is used to further eliminate the MUI. Given the SINR-based greedy beam selection, we design a CGA to solve the second subproblem, namely, the optimal deployment of the mmWave UAV-BS system. Similarly, considering the mobility of the mmWave 
TABLE 1: Reference comparison of recent researches.

\begin{tabular}{|c|c|c|c|c|c|c|c|c|c|c|c|c|c|}
\hline & $\begin{array}{c}{[7]} \\
2016\end{array}$ & $\begin{array}{c}{[8]} \\
2019\end{array}$ & $\begin{array}{c}{[9]} \\
2017\end{array}$ & $\begin{array}{c}{[10]} \\
2018\end{array}$ & $\begin{array}{c}{[11]} \\
2018\end{array}$ & $\begin{array}{c}{[12]} \\
2019\end{array}$ & $\begin{array}{c}{[18]} \\
2019\end{array}$ & $\begin{array}{c}{[20]} \\
2018\end{array}$ & $\begin{array}{c}{[21]} \\
2020\end{array}$ & $\begin{array}{c}{[25]} \\
2016\end{array}$ & $\begin{array}{r}{[26]} \\
2015\end{array}$ & $\begin{array}{r}{[28]} \\
2018\end{array}$ & $\begin{array}{r}{[33]} \\
2019\end{array}$ \\
\hline UAV-BS & $\sqrt{ }$ & $\sqrt{ }$ & $\sqrt{ }$ & $\sqrt{ }$ & $\sqrt{ }$ & $\sqrt{ }$ & $\sqrt{ }$ & $\sqrt{ }$ & $\sqrt{ }$ & & & & \\
\hline Deployment & $\sqrt{ }$ & $\sqrt{ }$ & $\sqrt{ }$ & & & & & & $\sqrt{ }$ & & & & \\
\hline 3D deployment & & & $\sqrt{ }$ & & & & $\sqrt{ }$ & $\sqrt{ }$ & & & & & $\sqrt{ }$ \\
\hline Trajectory & & & & $\sqrt{ }$ & $\sqrt{ }$ & $\sqrt{ }$ & & & & & & & \\
\hline mmWave & & & & & & & $\sqrt{ }$ & $\sqrt{ }$ & $\sqrt{ }$ & $\sqrt{ }$ & $\sqrt{ }$ & & \\
\hline Beamforming & & & & & & $\sqrt{ }$ & $\sqrt{ }$ & & $\sqrt{ }$ & $\sqrt{ }$ & $\sqrt{ }$ & & \\
\hline BDMA & & & & & & & & & & $\sqrt{ }$ & $\sqrt{ }$ & $\sqrt{ }$ & \\
\hline $\mathrm{ZF}$ & & & & & & $\sqrt{ }$ & & & & $\sqrt{ }$ & $\sqrt{ }$ & & \\
\hline Beam selection & & & & & & & & & & $\sqrt{ }$ & $\sqrt{ }$ & $\sqrt{ }$ & \\
\hline SINR & & & & $\sqrt{ }$ & & $\sqrt{ }$ & & & & & $\sqrt{ }$ & & \\
\hline Multiuser & & $\sqrt{ }$ & $\sqrt{ }$ & $\sqrt{ }$ & $\sqrt{ }$ & $\sqrt{ }$ & $\sqrt{ }$ & $\sqrt{ }$ & $\sqrt{ }$ & $\sqrt{ }$ & $\sqrt{ }$ & $\sqrt{ }$ & $\sqrt{ }$ \\
\hline Multiuser interference & & & & & & $\sqrt{ }$ & & & & $\sqrt{ }$ & $\sqrt{ }$ & & \\
\hline GA & & & & & & & & & & & & & $\sqrt{ }$ \\
\hline
\end{tabular}

UAV-BS, we also optimize the trajectory of the mmWave UAV-BS by the CGA scheme, given the SINR-based greedy beam selection. The main contributions of this paper are recapped below.

(1) In this paper, we consider a mmWave UAV-BS, which is equipped with the lens antenna array to serve the multiple ground users. Aimed at maximizing the sum rate of all ground users, the optimization problem of beam selection and UAV deployment is complicated to solve, which is decomposed into two subproblems

(2) First, we propose an SINR-based greedy beam selection scheme for the users which share the same dominant beams, where ZF precoding is employed to further minimize the MUI. Then, the CGA of UAV-BS deployment is designed to optimize the UAV deployment and beam pattern by maximizing the sum rate of ground users

(3) We also formulate an optimization problem of beam selection and UAV-BS trajectory in the multiuser mmWave system to maximize the sum rate of all ground users with the speed constraint of UAV-BS. Given the suboptimal SINR-based greedy beam selection, the CGA-based mmWave UAV-BS trajectory optimization is proposed

The paper is structured as follows. Section 2 introduces the system model and problem formulation. The optimizations of beam selection in beamspace as well as mmWave UAV-BS deployment and trajectory are addressed in Section 3. The design analysis and achievable performance are provided and discussed in Section 4. Section 5 concludes the paper.

In this paper, $\mathbb{R}$ and $\mathbb{C}$ denote the real and complex number fields, respectively. The boldfaced lowercase and uppercase letters stand for vectors and matrices, respectively. $\|\cdot\|$ denotes the Euclidean norm, while the transpose and Hermitian trans- pose operators are denoted by $(\cdot)^{\mathrm{T}}$ and $(\cdot)^{\mathrm{H}}$, respectively. The inverse operation is denoted by $(\cdot)^{-1}$, while $\operatorname{tr}(\cdot)$ represents the trace operation. The operator $\mathscr{A} \backslash \mathscr{B}$ denotes the set consisting of all elements in $\mathscr{A}$ excluding those in $\mathscr{B}$. The integer ceiling operation is denoted by $[\cdot], \mathbf{0}_{K}$ is the $K$-dimensional zero vector, and $\mathbf{I}_{K}$ is the $(K \times K)$-dimensional identity matrix, while $\mathbf{A}(i,:)_{i \in \mathscr{I}}$ denotes the submatrix consisting of the rows of $\mathbf{A}$ with the row indices given by the index set $\mathscr{F}$. The cardinality of the set $\mathscr{I}$ is denoted by $|\mathscr{I}|$, and the $j$ th element of $\mathscr{I}$ is denoted as $\mathscr{I}(j)$, while $\mathbf{w}_{(k,:)}$ and $\mathbf{w}_{(:, k)}$ denote the $k$ th row and $k$ th column of $\mathbf{W}$, respectively.

\section{System Model and Problem Formulation}

As illustrated in Figure 1, we consider an mmWave downlink multiuser communication system, where a rotary-wing UAVBS employs $N_{\mathrm{t}}$ transmit antennas and $N_{\mathrm{RF}}$ RF chains to simultaneously communicate with $K$ terrestrial users. The different users are served by different beams. Each ground user is equipped with a single antenna, and the horizontal coordinate of the $k$ th ground user is given by $\mathbf{u}_{k}=\left[x_{k} y_{k}\right]^{\mathrm{T}} \in \mathbb{R}^{2 \times 1}, 1 \leq k$ $\leq K$. We assume that the UAV-BS flies at a constant altitude $H(\mathrm{~m})$ above the ground and the location of UAV-BS projected on the ground in the $3 \mathrm{D}$ rectangular coordinate system is defined by $\mathbf{q}=\left[q_{x} q_{y}\right]^{\mathrm{T}} \in \mathbb{R}^{2 \times 1}$.

2.1. Channel Model. Owing to the flexibility of the UAV-BS, the LoS can be naturally established in low-altitude platforms [34], and scattering is relatively rare in mmWave frequencies for the air-to-ground links. Thus, we assume that the wireless links between the UAV-BS and the ground users are dominant by the LoS paths $[18,35]$. Then, the effective channel model between the UAV-BS and the $k$ th ground user can be expressed as [36]

$$
\mathbf{h}_{k}=\beta_{k} \mathbf{a}\left(\theta_{k}\right),
$$




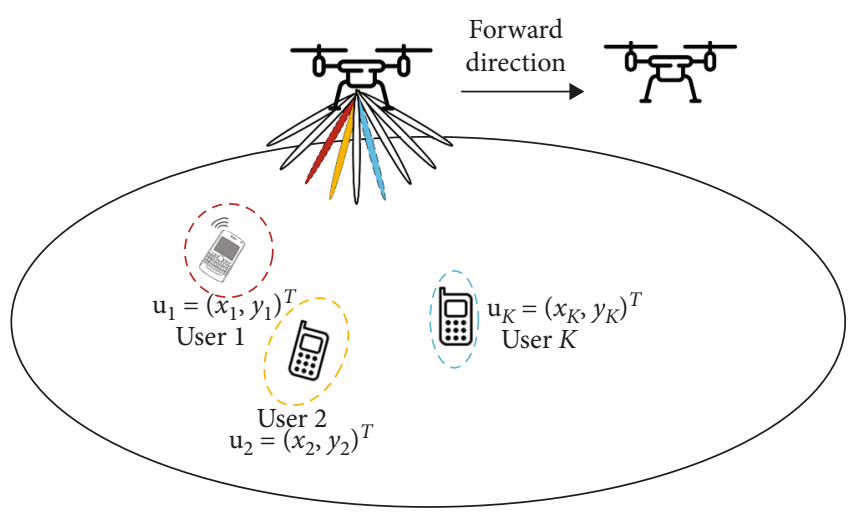

Figure 1: A mmWave UAV-BS serving multiple ground users.

where $\mathbf{a}\left(\theta_{k}\right) \in \mathbb{C}^{N_{\mathrm{t}} \times 1}$ is the array steering vector of the $k$ th ground user and $\beta_{k}$ is the gain of the LoS path for this ground user. The channel coefficient $\beta_{k}$ depends on the path loss at the mmWave frequency and is given by $[37,38]$

$$
\beta_{k}=\frac{1}{\left(4 \pi f_{m, m} \times 10^{9} / c\right) \cdot d_{k}^{\alpha_{\mathrm{L}}}}
$$

where $d_{k}=\sqrt{H^{2}+\left\|\mathbf{q}-\mathbf{u}_{k}\right\|^{2}}$ is the distance between the UAV-BS and the $k$ th ground user, $c=3 \times 10^{8} \mathrm{~m} / \mathrm{s}$ is the speed of light, $f_{m, m}$ is the carrier frequency, and $\alpha_{\mathrm{L}}$ is the parameter of the LoS path loss model. In our investigation, $f_{m, m}=28 \mathrm{GHz}$ is allocated and $\alpha_{\mathrm{L}}=0.95$ is adopted. In addition, the spatial direction $\theta_{k}$ is defined as $\theta_{k}=\cos \left(\phi_{k}\right)$, where $\phi_{k}$ is the real angle of departure of the $k$ th ground user. Hence, the range of $\theta_{k}$ is $[-1,1]$. A uniform linear array (ULA) is employed in the mmWave UAV-BS, and the array steering vector for the $k$ th ground user is expressed as [26]

$\mathbf{a}\left(\theta_{k}\right)=\left[e^{-\mathrm{j} 2 \pi \frac{d}{\lambda}\left(\frac{1-N_{\mathrm{t}}}{2}\right) \theta_{k}} \cdots e^{-\mathrm{j} 2 \pi \frac{d}{\lambda}\left(l-\frac{N_{\mathrm{t}}-1}{2}\right) \theta_{k}} \cdots e^{-\mathrm{j} 2 \pi \frac{d}{\lambda}\left(\frac{N_{\mathrm{t}}-1}{2}\right) \theta_{k}}\right]^{T}, l=0,1, \cdots N_{\mathrm{t}}-1$,

where $\lambda$ is the signal wavelength and $d=\lambda / 2$ is the antenna element spacing.

2.1.1. UAV-BS Deployment. For the mmWave UAV-BS deployment, the spatial direction of the $k$ th user $\theta_{k}$ is related to the placement of the UAV-BS, given as the cosine value of the direction vector from the $k$ th ground user to the UAVBS and the UAV-BS forward direction vector $[100]^{T}$ [21]. Thus, the spatial direction $\theta_{k}$ is defined as

$$
\begin{aligned}
\theta_{k} & =\frac{\left(\left[x_{k} y_{k} 0\right]-\left[q_{x} q_{y} H\right]\right) \cdot[100]^{T}}{\left\|\left[x_{k} y_{k} 0\right]^{T}-\left[q_{x} q_{y} H\right]^{T}\right\| \cdot\left\|[100]^{T}\right\|} \\
& =\frac{x_{k}-q_{x}}{\sqrt{\left(x_{k}-q_{x}\right)^{2}+\left(y_{k}-q_{y}\right)^{2}+H^{2}}} .
\end{aligned}
$$

2.1.2. UAV-BS Trajectory. To take into account the mobile nature of the UAV-BS, the UAV-BS trajectory should be considered. Specifically, the fly-hover communication protocol [39] of rotary-wing UAV-BS can be adopted to realize the communication with users. The UAV-BS serves the ground users with a constant mission time $T$, which can be equally divided into $N+1$ time slots. For this fly-hover communication protocol, the UAV-BS successively visits the $N$ optimized hovering locations and communicates with the ground users when it is hovering at the optimized locations. Since the UAV-BS flies at a constant altitude $H$, the horizontal coordinate in the $n$th time slot is defined as $\mathbf{q}[n]$ $=\left[q_{x}[n] q_{y}[n]\right]^{T}, n=0,1, \cdots, N+1$. In practice, the trajectory of the UAV-BS should satisfy the following constraints:

$$
\mathscr{A}_{\mathrm{tra}}=\left\{\begin{array}{l}
\mathbf{q}[0]=\mathbf{q}_{\mathrm{I}}, \mathbf{q}[N+1]=\mathbf{q}_{\mathrm{F}}, \\
\|\mathbf{q}[n+1]-\mathbf{q}[n]\| \leq v_{\max }, 0 \leq n \leq N, \\
\mathbf{q}[n] \in\left[q_{x, l}, q_{x, h}\right] \times\left[q_{y, l}, q_{y, h}\right], \forall n,
\end{array}\right.
$$

where $\mathbf{q}_{\mathrm{I}}, \mathbf{q}_{\mathrm{F}} \in \mathbb{R}^{2 \times 1}$ denote the initial and final horizontal locations of the UAV-BS, respectively, and $v_{\max }$ is the maximum speed of the UAV-BS, that is, the UAV-BS velocity $v \leq v_{\max }$, while $\left[q_{x, l}, q_{x, h}\right] \times\left[q_{y, l}, q_{y, h}\right]$ denotes the UAV-BS flying area. The forward direction vector of the UAV-BS is related to the location of the next time slot and is defined as $\mathbf{q}_{f}[n]=\left[\left(q_{x}[n+1]-q_{x}[n]\right)\left(q_{y}[n+1]-q_{y}[n]\right) 0\right]^{T}, n=0,1$, $\cdots, N$. Thus, the spatial direction of the $k$ th user at the $n$th time slot can be expressed as

$$
\theta_{k, n}=\frac{\left(\left[x_{k} y_{k} 0\right]-\left[q_{x} q_{y} H\right]\right) \cdot \mathbf{q}_{f}[n]}{\left\|\left[x_{k} y_{k} 0\right]^{T}-\left[q_{x} q_{y} H\right]^{T}\right\| \cdot\left\|\mathbf{q}_{f}[n]\right\|} .
$$

2.2. Beamspace Representation. By employing an elaborately designed DLA, the traditional channel (1) in the spatial domain can be translated into an equivalent one in the beamspace or angular domain [40]. In particular, let $\mathbf{U} \in$ $\mathbb{C}^{N_{\mathrm{t}} \times N_{\mathrm{t}}}$ be the spatial discrete Fourier transform (DFT) matrix representing the operation of the DLA, which is acquired by the steering vectors at the $N_{\mathrm{t}}$ fixed spatial frequencies with uniform spacing $[41,42]$. Thus, the DFT matrix $\mathbf{U}$, which contains the $N_{t}$ orthogonal beams that cover the whole space, is defined as

$$
\mathbf{U}=\frac{1}{\sqrt{N_{\mathrm{t}}}}\left[\mathbf{a}\left(\psi_{1}\right) \mathbf{a}\left(\psi_{2}\right) \cdots \mathbf{a}\left(\psi_{N_{t}}\right)\right]
$$

where $\psi_{n}=1 / N_{\mathrm{t}}\left(n-\left(\left(N_{\mathrm{t}}+1\right) / 2\right)\right), n=1,2, \cdots, N_{t}$, are the predefined spatial directions. The DFT matrix $\mathbf{U}$ forms the basis of the $N_{\mathrm{t}}$-dimensional spatial orthogonal signal space.

Let $\mathbf{x} \in \mathbb{C}^{K \times 1}$ be the transmitted symbol vector to the $K$ ground users and $y \in \mathbb{C}^{K \times 1}$ be the received signal vector at the $K$ ground users. Then, the system model in the beamspace domain can be expressed as 


$$
\mathbf{y}=\tilde{\mathbf{H}}^{H} \mathbf{W} \mathbf{x}+\mathbf{n},
$$

where $\mathbf{n} \sim \mathscr{C} \mathscr{N}\left(\mathbf{0}_{k}, \sigma_{n}^{2} \mathbf{I}_{K}\right)$ is the additive white Gaussian noise (AWGN) vector with the covariance matrix $\sigma_{n}^{2} \mathbf{I}_{K}, \mathbf{W} \in \mathbb{C}^{N_{\mathrm{t}} \times K}$ is the precoding matrix, and the beamspace channel $\tilde{\mathbf{H}} \in$ $\mathbb{C}^{N_{\mathrm{t}} \times K}$ is defined as

$$
\tilde{\mathbf{H}}=\left[\mathbf{h}_{b, 1} \mathbf{h}_{b, 2} \cdots \mathbf{h}_{b, K}\right]=\left[\mathbf{U h}_{1} \mathbf{U h}_{2} \cdots \mathbf{U h}_{K}\right]=\mathbf{U H},
$$

where $\mathbf{H} \in \mathbb{C}^{N_{\mathrm{t}} \times K}$ is the spatial channel for all the $K$ users and $\mathbf{h}_{b, k} \in \mathbb{C}^{N_{\mathrm{t}} \times 1}$ is the beamspace channel of the $k$ th user. The beamspace channel $\tilde{\mathbf{H}}$ indicates that the signals of each user are mapped in the $N_{\mathrm{t}}$ orthogonal beams, and each element of $\mathbf{h}_{b, k}$ denotes one of the $N_{\mathrm{t}}$ beams supported by the DLA.

\subsection{Problem Formulation}

2.3.1. UAV-BS Deployment Problem Formulation. Let the UAV hovering area $\mathscr{A}_{\text {dep }}$ be defined by

$$
q_{x, l} \leq q_{x} \leq q_{x, u}, q_{y, l} \leq q_{y} \leq q_{y, u}
$$

where $q_{x, l}$ and $q_{y, l}$ denote the minimum values of $q_{x}$ and $q_{y}$, respectively, while $q_{x, u}$ and $q_{y, u}$ represent the maximum values of $q_{x}$ and $q_{y}$, respectively. The UAV-BS deployment problem is to determine the position $\mathbf{q} \in \mathscr{A}_{\text {dep }}$ to deploy the UAV-BS. Due to the sparse nature of the beamspace channel [24], there are only a few dominant entries of the beamspace channel for each user. The use of the beamspace channel allows us to select the corresponding beams to reduce the dimension of the mmWave UAV-BS system without significant performance loss. The reduced system model in the beamspace domain is given by

$$
\tilde{\mathbf{y}}=\tilde{\mathbf{H}}_{r}^{H} \mathbf{W}_{r} \mathbf{x}+\mathbf{n},
$$

where $\tilde{\mathbf{H}}_{r}=\tilde{\mathbf{H}}(b,:)_{b \in \mathscr{B}}$ and $\mathscr{B}$ consists of the indices of the selected beams, while $\mathbf{W}_{r}$ is the precoding matrix with the corresponding reduced dimension. Thus, the received signal at the $k$ th user can be expressed as

$$
\tilde{y}_{k}=\tilde{\mathbf{h}}_{r(k,:)}^{H} \mathbf{w}_{r(:, k)}^{\sim} x_{k}+\sum_{j=1, j \neq k}^{K} \tilde{\mathbf{h}}_{r(k,:)}^{H} \mathbf{w}_{r(:, j)}^{\sim} x_{j}+n_{k},
$$

where $\tilde{y}_{k}$ is the $k$ th element of $\tilde{\mathbf{y}}$. Based on the above discussion, for the UAV-BS transmission with multiple users, the average achievable rate of the $k$ th user is

$R_{k}=\log _{2}\left(1+\left(P_{\mathrm{t}} / K\right) \| \frac{\tilde{\mathbf{h}}_{r(k,:)}^{H} \mathbf{w}_{r(:, k)}^{\sim} \|^{2}}{\left.\sigma_{n}^{2}+\left(P_{\mathrm{t}} / K\right) \sum_{j=1, j \neq k}^{K}\left\|\tilde{\mathbf{h}}_{r(k,:)}^{H} \mathbf{w}_{r(:, j)}^{\sim}\right\|^{2}\right),}\right.$

where $P_{\mathrm{t}}$ is the total transmission power of the UAV-BS. Thus, the sum rate of all the users is $R_{\text {sum }}=\sum_{k=1}^{K} R_{k}$.

Note that the beamspace channel $\tilde{\mathbf{H}}$ is a function of the UAV-BS position $\mathbf{q}$, and hence, $\mathscr{B}$ also depends on $\mathbf{q}$ as well as the way of beam selection. Therefore, $R_{\text {sum }}$ depends on both $\mathbf{q}$ and $\mathscr{B}$, and the UAV-BS placement problem in the beamspace domain can be formulated as

$$
\begin{gathered}
\max _{\mathbf{q}, \mathscr{B}} R_{\text {sum }}(\mathbf{q}, \mathscr{B}), \\
\text { s.t. }
\end{gathered}
$$

2.3.2. UAV-BS Trajectory Problem Formulation. The UAVBS trajectory problem is to determine the mission trajectory $\{\mathbf{q}[n]\}_{n=0}^{N} \in \mathscr{A}_{\text {tra }}$ of the UAV-BS. At the $n$th time slot, given the UAV-BS position $\mathbf{q}[n]$, the beamspace channel $\tilde{\mathbf{H}}$ is known. Let $\mathscr{B}[n]$ be the corresponding selected beam set. In order to study the performance of the UAV-BS trajectory, we consider the sum rate of the $k$ th user served by the UAVBS in the $n$th time slot of a mission, which is expressed as

$R_{k}[n]=\log _{2}\left(1+\left(P_{\mathrm{t} 1} / K\right) \| \frac{\tilde{\mathbf{h}}_{r(k,:)}^{H}[n] \mathbf{w}_{r(:, k)}^{\sim}[n] \|^{2}}{\left.\sigma_{n}^{2}+\left(P_{\mathrm{t} 1} / K\right) \sum_{j=1, j \neq k}^{K}\left\|\tilde{\mathbf{h}}_{r(k,:)}^{H}[n] \mathbf{w}_{r(:, j)}^{\sim}[n]\right\|^{2}\right),}\right.$

where $\tilde{\mathbf{h}}_{r(k,:)}[n]$ is the reduced beamspace channel vector of the $k$ th user in the $n$th time slot, $P_{\mathrm{t} l}$ denotes the transmit power at the UAV-BS for each time slot, and $\mathbf{w}_{r(:, k)}^{\sim}[n]$ is the reduced precoding vector of the $k$ th user in time slot $n$. Therefore, the sum rate for all the users over the $N+1$ time slots can be expressed as $R_{\text {sum }, N}=\sum_{k=1}^{K} \sum_{n=0}^{N} R_{k}[n]$.

Based on the above discussion, we can formulate the sum rate maximization problem for the UAV-BS trajectory as

$\max _{\{\mathbf{q}[n]\}_{n=0}^{N},\{\mathscr{B}[n]\}_{n=1}^{N}} R_{\text {sum }, N}\left(\{\mathbf{q}[n]\}_{n=0}^{N},\{\mathscr{B}[n]\}_{n=1}^{N}\right)$,

$$
\text { s.t. } \quad\{\mathbf{q}[n]\}_{n=0}^{N} \in \mathscr{A}_{\text {tra }} \text {. }
$$

$\{B[n]\}_{n=1}^{N}$ depends on the UAV-BS trajectory $\{\mathbf{q}[n]\}_{n=1}^{N}$ and how the beams are selected at each time slot $n$.

\section{Solution of the Problem}

By evaluating the Hessian matrices of the objective functions for the problems (14) and (16), it can be seen that both the optimization problems are nonconvex and highly complex. Therefore, it is challenging to solve the problems (14) and (16) by the existing optimization tools. We focus on the optimization problem of UAV-BS deployment first by separating the problem (14) into two subproblems. The "inner" subproblem determines the selected beam set $B$ giving the UAV-BS position q. A suboptimal SINR beam selection algorithm is introduced to incrementally maximize the sum rate of the mmWave UAV-BS system. With this SINR beam selection scheme, the "outer" subproblem then solves the UAV-BS deployment by employing the CGA algorithm. 
Next, we solve the optimization problem (16) for UAV-BS trajectory in a similar way. Specifically, the CGA is designed to optimize the UAV-BS trajectory with the proposed suboptimal SINR beam selection scheme.

3.1. Beam Selection. Given the UAV-BS position $\mathbf{q}$, the beamspace channel $\tilde{\mathbf{H}}$ is known. The proposed SINR beam selection scheme is composed of two parts. The ground users having unique dominant beams can select the dominant beams to communicate with the UAV-BS, while the users having overlap dominant beams will search for the proper beams from the unselected beams by incrementally maximizing the SINR of the mmWave UAV-BS system, where $\mathrm{ZF}$ is utilized to further eliminate the MUI.

Due to the sparse structure of the beamspace channel in the mmWave UAV-BS system, the beamspace channel for the $k$ th user has dominant values near the LoS direction [25]. Since the dominant beams have the most transmission power, they can be selected for the transmission to reduce the number of RF chains with minor performance loss. In order to reduce the number of RF chains $N_{\mathrm{RF}}$ to the minimum, we consider that each user is served by only one beam, and hence, $N_{\mathrm{RF}}=K$. Let the index of the selected dominant beam for user $k$ be $\mathscr{B}_{\max , k}$, which is defined as [43]

$$
\mathscr{B}_{\max , k}=\left\{i^{\star}=\arg \max _{1 \leq i \leq N_{t}}\left|h_{b, k}^{i}\right|^{2}\right\},
$$

where $h_{b, k}^{i}$ denotes the $i$ th element of the $k$ th column of the beamspace channel $\tilde{\mathbf{H}}$.

Given the beamspace channel vectors $\mathbf{h}_{b, k}$ and the corresponding selected beam indexes (17) for $1 \leq k \leq K$, the $K$ users can be assigned into the two groups, the nonoverlap group $\mathscr{G}_{\mathrm{N}}$ and the overlap groups $\mathscr{G}_{\mathrm{O}}$. The group $\mathscr{G}_{\mathrm{N}}$ includes the users having the unique dominant beams, while a user in the group $\mathscr{G}_{\mathrm{O}}$ has the same selected beam with some other users in $\mathscr{G}_{\mathrm{O}}$. In particular, the selected beam set or index of a nonoverlap user $i$ satisfies $\mathscr{B}_{\max , i} \cap$ $\left(\cup_{j \neq i, 1 \leq j \leq K} \mathscr{B}_{\max , j}\right)=\varnothing$. On the other hand, if $\mathscr{B}_{\max , i}{ }^{\prime} \cap$

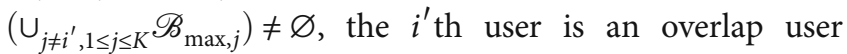
and is assigned to $\mathscr{G}_{\mathrm{O}}$. Obviously, $\mathscr{G}_{\mathrm{N}}$ and $\mathscr{G}_{\mathrm{O}}$ satisfy $\mathscr{G}_{\mathrm{N}} \cup \mathscr{G}_{\mathrm{O}}=\{1,2, \cdots, K\}$ and $\mathscr{G}_{\mathrm{O}} \cap \mathscr{G}_{\mathrm{N}}=\varnothing$. We consider an example of $K=6$ and $N_{\mathrm{t}}=8$, where $\mathscr{B}_{\max , 1}=\{1\}, \mathscr{B}_{\max , 2}=$ $\{4\}, \mathscr{B}_{\max , 3}=\{7\}, \mathscr{B}_{\max , 4}=\{7\}, \mathscr{B}_{\max , 5}=\{8\}$, and $\mathscr{B}_{\max , 6}$ $=\{8\}$. Then, $\mathscr{G}_{\mathrm{N}}=\{1,2\}$ and $\mathscr{G}_{\mathrm{O}}=\{3,4,5,6\}$.

For nonoverlap user $k \in \mathscr{G}_{N}$, the beam with index $\mathscr{B}_{\max , k}$ will be directly selected, since this dominant beam contains most of the transmission power and causes few interferences to other users. Let $\mathscr{B}_{\mathscr{G}_{\mathrm{N}}}$ denote the selected beams for the nonoverlap users in $\mathscr{G}_{\mathrm{N}}$. For the overlap users in $\mathscr{G}_{\mathrm{O}}$, the beams should be reselected to communicate with the UAV-BS by maximizing the sum rate of all users. To incrementally maximize the sum rate, the beams which result in the maximum increase in SINR should be selected. In order to mitigate the MUI with the user group $\mathscr{G}_{\mathrm{N}}$, the beams for the users in $\mathscr{G}_{\mathrm{O}}$ are selected from the beam set $\mathscr{B}_{R}=\{1,2$, $\left.\cdots, N_{\mathrm{t}}\right\} \backslash \mathscr{B}_{\mathscr{G}_{\mathrm{N}}}$. Let $\mathscr{B}_{\mathscr{G}_{\mathrm{O}}}$ denote the selected beams for the overlap users in $\mathscr{G}_{\mathrm{O}}$.

The precoding matrix $\mathbf{W}_{r}$ for all the users with the selected beams $\mathscr{B}_{\text {sel }}=\mathscr{B}_{\mathscr{G}_{\mathrm{O}}} \cup \mathscr{B}_{\mathscr{G}_{\mathrm{N}}}$ can be expressed as

$$
\mathbf{W}_{r}=\alpha \mathbf{F}_{r}
$$

where $\alpha$ is the power normalization factor given by

$$
\alpha=\sqrt{\frac{P_{\mathrm{t}}}{\operatorname{tr}\left(\boldsymbol{F}_{r} \boldsymbol{F}_{r}^{H}\right)}},
$$

and $\mathbf{F}_{r}$ is the $\mathrm{ZF}$ precoding matrix expressed as

$$
\mathbf{F}_{r}=\tilde{\mathbf{H}}_{r}\left(\tilde{\mathbf{H}}_{r}^{H} \tilde{\mathbf{H}}_{r}\right)^{-1}
$$

in which $\tilde{\mathbf{H}}_{r}=\tilde{\mathbf{H}}(b,:)_{b \in \mathscr{B}_{\text {sel }}}$. The SINR with the ZF precoding for user $k$ can be expressed as [44]

$$
\operatorname{SINR}_{k, Z F}=\frac{P_{\mathrm{t}}|\alpha|^{2}}{K \sigma_{n}^{2}} .
$$

Thus, the maximization of the SINR can be transformed to the maximization of the normalization factor $\alpha$ with respect to the selected beam set $\mathscr{B}_{\mathscr{G}_{\mathrm{O}}}$ for the overlap users in $\mathscr{G}_{\mathrm{O}}$.

Hence, we can select the required beams for the overlap users in $\mathscr{G}_{\mathrm{O}}$ one by one based on a greedy-type beam selection algorithm that identifies the beams with the maximal gains in terms of SINR. Specifically, at each selection stage, a beam is selected for an overlap user in $\mathscr{G}_{\mathrm{O}}$ which maximizes the gain in the SINR. This greedy beam selection algorithm is summarized in Algorithm 1.

3.2. Continuous Genetic Algorithm for UAV-BS Deployment. For given q, we use Algorithm 1 to select the beam set $\mathscr{B}_{\text {dep }}(\mathbf{q})=\mathscr{B}_{\text {sel }}(\mathbf{q})$. Express the objective function of the UAV-BS deployment problem for this beam selection scheme as $f_{\text {dep }}(\mathbf{q})=R_{\text {sum }}\left(\mathbf{q}, \mathscr{B}_{\text {dep }}(\mathbf{q})\right)$. Hence, with this greedy SINR-based beam selection scheme, the optimization of the UAV-BS deployment can be expressed as

$$
\mathbf{q}^{\star}=\arg \max _{\mathbf{q} \in \mathscr{A}_{\mathrm{dep}}} f_{\mathrm{dep}}(\mathbf{q}) \text {. }
$$

We propose to apply the CGA $[30,45,46]$ to solve this optimization problem. With $P_{\text {dep }}$ denoting the population size and $g$ being the generation index, the procedure of the CGA-based UAV-BS deployment is detailed below.

(1) Initialization: at $g=0$, the initial population of $P_{\text {dep }}$ chromosomes or candidate UAV-BS locations $\left\{\mathbf{q}_{g, p}\right\}_{p=1}^{P_{\text {dep }}}$ are randomly generated over the UAV hovering area $\mathscr{A}_{\text {dep }}$. Algorithm 1 is utilized to select the beam set $\mathscr{B}_{\text {dep }}\left(\mathbf{q}_{g, p}\right)$ for every chromosome $\mathbf{q}_{g, p}$ 


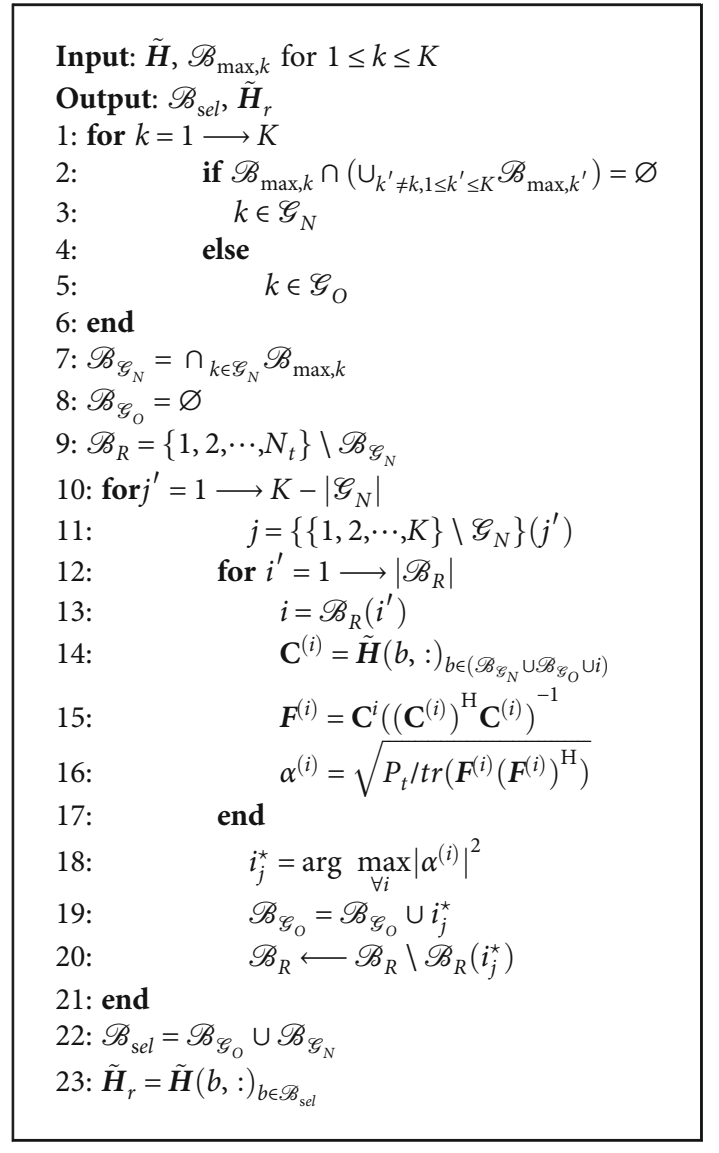

Algorithm 1: Greedy SINR-based beam selection.

, and then, the fitness value $F_{\operatorname{dep}_{g, p}}=f_{\text {dep }}\left(\mathbf{q}_{g, p}\right)$ is calculated, for $1 \leq p \leq P_{\text {dep }}$. The $P_{\text {dep }}$ chromosomes are ranked from top to bottom according to their fitness values. Denote this ranked population as $\left\{\mathbf{q}_{g, p} ; F_{\operatorname{dep}_{g, p}}\right\}_{p=1}^{P_{\text {dep }}}$ with the fitness values $F_{\text {dep }_{g, 1}} \geq$ $F_{\operatorname{dep}_{g, 2}} \geq \cdots \geq F_{\text {dep }_{g, P_{\text {dep }}}}$

(2) Selection: set $g=g+1$. The $P_{\text {keep }, d}$ previous chromosomes with the highest fitness values will survive into the next generation, i.e., $\left\{\mathbf{q}_{g, p}=\mathbf{q}_{g-1, p} ; F_{\operatorname{dep}_{g, p}}=F_{\operatorname{dep}_{g-1, p}}\right\}_{p=1}^{P_{\text {keep }, d}}$, and these high-fitness chromosomes also form a mating pool, where $P_{\text {keep }, d}=P_{\text {dep }} \cdot \gamma_{\text {dep }}$ is the size of the mating pool. The selection ratio $\gamma_{\text {dep }}$ decides how many chromosomes are to be chosen for the mating pool from the total $P_{\text {dep }}$ chromosomes. The mating pool is used to select parents to provide $P_{\text {dep }}-P_{\text {keep }, d}$ offspring for the next generation to maintain a constant-size population

The rank weighting, a roulette wheel weighting [30], is used to choose chromosomes for mating. With this weighting, the chromosome with the largest fitness has the greatest probability of mating, while the chromosome with the smal- lest fitness has the lowest probability of mating. Let $P_{m}^{\text {dep }}$ be the probability of selecting the $m$ th chromosome for mating from the $P_{\text {keep, } d}$ chromosomes, which is calculated as

$$
P_{m}^{\mathrm{dep}}=\frac{P_{\text {keep }, d}-m+1}{\sum_{m=1}^{P_{\text {keep }, d}} m}, 1 \leq m \leq P_{\text {keep }, d} .
$$

Then, $P_{m}^{\mathrm{dep}}$ is turned into the cumulative probability $P_{c, m}^{\mathrm{dep}}$ for deciding which chromosome can be selected as a parent. In particular, when a random number $P_{\text {mum }}$ between 0 and 1 is generated, if $P_{c, m-1}^{\mathrm{dep}}<P_{\text {mum }} \leq P_{c, m}^{\mathrm{dep}}$, the $m$ th chromosome will be selected as a parent (mum). For instance, let $P_{\text {keep, } d}$ $=4$, and the corresponding $P_{m}^{\mathrm{dep}} \in\{0.4,0.3,0.2,0.1\}$. Then, $P_{c, m}^{\mathrm{dep}} \in\{0.4,0.7,0.9,1\}$. If $P_{\mathrm{mum}}=0.33$, the first chromosome is selected as "mum." Similarly, the "dad" chromosome is chosen in the same manner. We adopt two parents producing two offspring as a common form of mating. Thus, $P_{\text {dep }}$ $-P_{\text {keep }, d}$ parents are required for mating to create new offspring. We will assume that $P_{\text {dep }}-P_{\text {keep }, d}$ is an even number, and we have selected $\left(P_{\text {dep }}-P_{\text {keep }, d}\right) / 2$ pairs of parents.

(3) Crossover: each pair of parents selected for the crossover operation can be expressed as

$$
\left\{\begin{array}{l}
\mathbf{q}_{g, \text { mum }}=\left[q_{x, g, \text { mum }} q_{y, g, \text { mum }}\right]^{T}, \\
\mathbf{q}_{g, \text { dad }}=\left[q_{x, g, \text { dad }} q_{y, g, \text { dad }}\right]^{T}
\end{array}\right.
$$

They are used to produce two offspring $\mathbf{q}_{g, \text { os } 1}$ and $\mathbf{q}_{g, \text { os } 2}$. The single crossover point scheme is utilized to combine the parents' information. A crossover point $q_{p_{c}}$ is randomly selected between $x$ and $y$. If $y$ is selected as the crossover point, i.e., $q_{p_{c}}=y$, the two new offspring are produced as

$$
\left\{\begin{array}{l}
\mathbf{q}_{g, \mathrm{os} 1}=\left[q_{x, g, \mathrm{mum}} q_{p_{c}, \mathrm{~g}, \mathrm{os} 1}\right]^{T}, \\
\mathbf{q}_{g, \mathrm{os} 2}=\left[q_{x, \mathrm{~g}, \mathrm{dad}} q_{p_{c}, g, \mathrm{os} 2}\right]^{T},
\end{array}\right.
$$

with

$$
\left\{\begin{array}{l}
q_{p_{c}, g, \text { os } 1}=q_{p_{c}, g, \text { mum }}-\mu\left(q_{p_{c}, g \text { mum }}-q_{p_{c}, g, \mathrm{dad}}\right)=q_{y, g, \text { mum }}-\mu\left(q_{y, g, \text { mum }}-q_{y, g, \mathrm{dad}}\right), \\
q_{p_{c}, g, 0 \mathrm{~s} 2}=q_{p_{c}, g, \mathrm{dad}}+\mu\left(q_{p_{c}, g, \mathrm{mum}}-q_{p_{c}, g, \mathrm{dad}}\right)=q_{y, g, \mathrm{dad}}+\mu\left(q_{y, g, \mathrm{mum}}-q_{y, g, \mathrm{dad}}\right),
\end{array}\right.
$$

where $\mu$ is a random value uniformly selected in the range of $(0,1)$. Similarly, if $x$ is selected as the crossover point, i.e., $q_{p_{c}}=x$, the crossover operation produces the two corresponding offspring from the pair of parents.

Algorithm 1 selects the beam set for every offspring, and its fitness value is calculated. After the crossover, the new generation of the chromosomes include the $P_{\text {keep }, d}$ elitist 
chromosomes from the previous generation and their $P_{\text {dep }}$ $-P_{\text {keep }, d}$ offspring. These $P_{\text {dep }}$ chromosomes are ranked from top to bottom according to their fitness values as $\left\{\mathbf{q}_{g, p} ; F_{\text {dep }_{g, p}}\right\}_{p=1}^{P_{\text {dep }}}$.

(4) Mutation: the element $q_{p_{m}, g, p}$ of chromosome $\mathbf{q}_{g, p}$ is randomly selected to mutate; thereby, the mutated element is replaced by a new random value $q_{p_{m},} \leq$ $q_{p_{m}, g, p} \leq q_{p_{m}, u}$, where $p_{m}=x$ or $y$, while $2 \leq p \leq P_{\text {dep }}$. We opt to not mutate the best chromosome with the highest fitness found so far, i.e., $\mathbf{q}_{g, 1}$. The number of elements that will mutate in each generation is governed by a mutation probability $M_{\text {dep }}[45,46]$. Thus, the total number of mutated elements is 2 ( $\left.P_{\text {dep }}-1\right) M_{\text {dep }}$ since each chromosome has 2 elements. The mutation operation is crucial to providing sufficient diversity for the CGA

Algorithm 1 then selects the beam sets for the mutated chromosomes, and their fitness values are calculated. Afterward, the mutated population of the $P_{\text {dep }}$ chromosomes is ranked again from top to bottom according to their fitness values as $\left\{\mathbf{q}_{g, p} ; F_{\text {dep }_{g, p}}\right\}_{p=1}^{P_{\text {dep }}}$.

(5) Termination: if $g>G_{\max , \mathrm{dep}}$, where $G_{\text {max,dep }}$ is the maximum number of generations, the procedure stops and the chromosome with the highest fitness is chosen as the solution, i.e., $\mathbf{q}^{\star}=\mathbf{q}_{g, 1}$. Otherwise, the procedure repeats from step (2)

The CGA-based UAV-BS deployment algorithm is summarized in Algorithm 2. The population size $P_{\text {dep }}$, the maximum number of generations $G_{\text {max,dep }}$, the selection ratio $\gamma_{\text {dep }}$, and the mutation probability $M_{\text {dep }}$ are the problemdependent algorithmic parameters, which need to be carefully chosen.

3.3. Continuous Genetic Algorithm for UAV-BS Trajectory. With the initial and final locations $\mathbf{q}_{\mathrm{I}}$ and $\mathbf{q}_{\mathrm{F}}$ fixed, the UAV-BS trajectory has $N$ locations, which is expressed as

$$
\mathbf{q}_{N}=\left[\mathbf{q}^{T}[1] \mathbf{q}^{T}[2] \cdots \mathbf{q}^{T}[N]\right]^{T}
$$

For given $\boldsymbol{q}[n]$ at time slot $n$, we use Algorithm 1 to select the beam set $\mathscr{B}_{\text {tra }}(\mathbf{q}[n])=\mathscr{B}_{\text {sel }}(\mathbf{q}[n])$. Denote the overall beam set selected for $\mathbf{q}_{\mathrm{N}}$ by Algorithm 1 as $\mathscr{B}_{\text {tra }}\left(\mathbf{q}_{N}\right)=$ $\left\{\mathscr{B}_{\text {sel }}(\mathbf{q}[n])\right\}_{n=1}^{N}$. Then, the optimization of the UAV-BS trajectory is reduced to

$$
\mathbf{q}_{N}^{\star}=\arg \max _{\mathbf{q}_{N} \in \mathscr{A}_{\text {tra }}} f_{\text {tra }}\left(\mathbf{q}_{N}\right),
$$

where the objective function of the UAV-BS trajectory problem is $f_{\text {tra }}\left(\mathbf{q}_{N}\right)=R_{\text {sum }, N}\left(\mathbf{q}_{N}, \mathscr{B}_{\text {tra }}\left(\mathbf{q}_{N}\right)\right)$. We also apply the CGA to solve this optimization problem. From (27), each chromosome has $2 \mathrm{~N}$ coordinates, and the $p$ th chromosome in the $g$ th generation can be expressed as

$$
\begin{array}{r}
\mathbf{q}_{N_{g, p}}=\left\{\mathbf{q}_{g, p}[n]\right\}_{n=1}^{N}=\left[q_{x, g, p}[1] q_{y, g, p}[1] q_{x, g, p}[2] q_{y, g, p}[2]\right. \\
\left.\cdots q_{x, g, p}[N] q_{y, g, p}[N]\right]^{T} .
\end{array}
$$

With the population size $P_{\text {tra }}$, the procedure of the CGA for the UAV-BS trajectory is now detailed.

(1) Initialization: at $g=0$, the initial population of $P_{\text {tra }}$ chromosomes $\left\{\mathbf{q}_{N_{g, p}}\right\}_{p=1}^{P_{\text {tra }}}$ is randomly generated, constrained inside $\mathscr{A}_{\text {tra }}$. Algorithm 1 then selects the beam sets $\left\{\mathscr{B}_{\text {tra }}\left(\mathbf{q}_{N_{g, p}}\right)\right\}_{p=1}^{P_{\text {tra }}}$, and the corresponding fitness values $\left\{F_{\text {tra }_{g, p}}=f_{\text {tra }}\left(\mathbf{q}_{N_{g, p}}\right)\right\}_{p=1}^{P_{\text {tra }}}$ are calculated. Then, these $P_{\text {tra }}$ chromosomes are ranked from top to bottom according to their fitness values as $\left\{\mathbf{q}_{N_{g, p}} ; F_{\text {tra }_{g, p}}\right\}_{p=1}^{P_{\text {tra }}}$ with the fitness values $F_{\text {tra }_{g, 1}} \geq$ $F_{\text {tra }_{g, 2}} \geq \cdots \geq F_{\text {tra }_{g, P_{\text {tra }}}}$

(2) Selection: set $g=g+1$, and retain the $P_{\text {keep }, t}$ previous chromosomes with the highest fitness values in the next generation as $\left\{\mathbf{q}_{N_{g, p}}=\mathbf{q}_{N_{g-1, p}} ; F_{\text {tra }_{g, p}}=F_{\text {tra }_{g-1, p}}\right\}_{p=1}^{P_{\text {keep }, t}}$, which also form a mating pool, where $P_{\text {keep }, t}=P_{\text {tra }}$. $\gamma_{\text {tra }}$ with $\gamma_{\text {tra }}$ being the selection ratio. As usual, the mating pool is used to select parents to provide $P_{\text {tra }}$ $-P_{\text {keep }, t}$ offspring for the next generation to maintain a constant-size population

The rank weight is also adopted to select parents from the mating pool. The probability $P_{m}^{\text {tra }}$ of the $m$ th chromosome to be selected from the mating pool is again defined by

$$
P_{m}^{\text {tra }}=\frac{P_{\text {keep }, t}-m+1}{\sum_{m=1}^{P_{\text {keep }, t}} m}, \quad 1 \leq m \leq P_{\text {keep }, t},
$$

and $P_{m}^{\text {tra }}$ is converted into the cumulative probability $P_{c, m}^{\text {tra }}$ to choose chromosomes as parents from the mating pool.

(3) Crossover: the mating strategy with two parents producing two new offspring is utilized, and hence, a total of $\left(P_{\text {tra }}-P_{\text {keep }, t}\right) / 2$ pairs of parents are selected. A generic pair of two parents selected from the mating pool can be expressed as 


$$
\left\{\begin{array}{l}
\mathbf{q}_{N_{g, \text { mum }}}=\left[q_{x, g, \text { mum }}[1] q_{y, g, \text { mum }}[1] \cdots q_{x, g, \text { mum }}[n] q_{y, g, \text { mum }}[n] \cdots q_{x, g, \text { mum }}[N] q_{y, g, \text { mum }}[N]\right]^{T}, \\
\mathbf{q}_{N_{g, \text { dad }}}=\left[q_{x, g, \text { dad }}[1] q_{y, g, \text { dad }}[1] \cdots q_{x, g, \text { dad }}[n] q_{y, g, \text { dad }}[n] \cdots q_{x, g, \text { dad }}[N] q_{y, g, \text { dad }}[N]\right]^{T} .
\end{array}\right.
$$



Algorithm 2: Continuous GA for UAV-BS deployment.

The number of crossover points depends on the UAV-BS flying area $\left[q_{x, l} q_{x, h}\right] \times\left[q_{y, l} q_{y, h}\right]$ and the maximum speed constraint $v_{\max }$. In particular, the longest straight flying distance is the diagonal line $\sqrt{\left(q_{x, h}-q_{x, l}\right)^{2}+\left(q_{y, h}-q_{y, l}\right)^{2}}$; thereby, the minimum required number of time slots to fly across the UAV-BS flying area can be expressed as

$$
N_{l}=\left\lceil\frac{\sqrt{\left(q_{x, h}-q_{x, l}\right)^{2}+\left(q_{y, h}-q_{y, l}\right)^{2}}}{v_{\max }}\right\rceil-1
$$

Thus, the $2 N_{l}$-point crossover scheme is utilized to 
guarantee the offspring satisfying the constraints $\mathscr{A}_{\text {tra. }}$. Specifically, the first crossover point $n$ is randomly selected from $\left\{1,2, \cdots, N-N_{l}+1\right\}$, and the two offspring produced can be expressed, respectively, as

$$
\begin{aligned}
& \mathbf{q}_{N_{g, o s 1}}=\left[q_{x, g, \text { mum }}[1] q_{y, g, \text { mum }}[1] \cdots q_{x, g, \text { mum }}[n-1] q_{y, g, \text { mum }}[n-1]\right. \\
& \mid q_{x, g, \text { os } 1}[n] q_{y, g, \text { os } 1}[n] q_{x, g, \text { os } 1}[n+1] \cdots q_{x, g, \text { os } 1}\left[n+N_{l}-1\right] \\
& q_{y, g, \text { os } 1}\left[n+N_{l}-1\right] \mid q_{x, g, \text { dad }}\left[n+N_{l}\right] q_{y, g, \text { dad }}\left[n+N_{l}\right] \\
& \left.\cdots q_{x, g, \text { dad }}[N] q_{y, g, \text { dad }}[N]\right]^{\mathrm{T}}, \mathbf{q}_{N_{g, 0 s 2}} \\
& =\left[q_{x, g, \text { dad }}[1] q_{y, g, \text { dad }}[1] \cdots q_{x, g, \text { dad }}[n-1] q_{y, g, \text { dad }}[n-1]\right. \\
& \mid q_{x, g, \mathrm{os} 2}[n] q_{y, g, \mathrm{os} 2}[n] q_{x, g, \mathrm{os} 2}[n+1] \cdots q_{x, \mathrm{~g}, \mathrm{os} 2}\left[n+N_{l}-1\right] \\
& q_{y, g, \mathrm{os} 2}\left[n+N_{l}-1\right] \mid q_{x, g, \text { mum }}\left[n+N_{l}\right] q_{y, g, \text { mum }}\left[n+N_{l}\right] \\
& \left.\cdots q_{x, g, \text { mum }}[N] q_{y, g, \text { mum }}[N]\right]^{\mathrm{T}} \text {. }
\end{aligned}
$$

Each offspring contains portions of the "genes" from both parents. Specifically, offspring os1 has mum's genes before the first crossover point $n$ and dad's genes after the last crossover point $n+N_{l}-1$, while offspring os 2 has dad's genes before the first crossover point $n$ and mum's genes after the last crossover point $n+N_{l}-1$. The new coordinates $q_{x}$ and $q_{y}$ at the crossover points are the combinations of the coordinates from both parents, which can be expressed as

$$
\left\{\begin{array}{l}
q_{x / y, g, \mathrm{os} 1}\left[n_{l}\right]=q_{x / y, g, \mathrm{mum}}\left[n_{l}\right]-\mu\left(q_{x / y, g, \mathrm{mum}}\left[n_{l}\right]-q_{x / y, g, \mathrm{dad}}\left[n_{l}\right]\right), \\
q_{x l y, g, \mathrm{os} 2}\left[n_{l}\right]=q_{x l y, g, \mathrm{dad}}\left[n_{l}\right]+\mu\left(q_{x / y, g, \mathrm{mum}}\left[n_{l}\right]-q_{x / y, g, \mathrm{dad}}\left[n_{l}\right]\right),
\end{array}\right.
$$

for $n \leq n_{l} \leq n+N_{l}-1$, where $\mu$ is a random value uniformly selected from $(0,1)$. If the new coordinates produced do not satisfy the maximum speed constraint of $\mathscr{A}_{\text {tra }}, \mu$ is reselected until the new coordinates meet the requirements of $\mathscr{A}_{\text {tra }}$.

The beam set of every offspring is then selected using Algorithm 1, and its fitness value is calculated. The new generation of the chromosomes, including the $P_{\text {keep }, t}$ elitist chromosomes from the previous generation and their $P_{\text {tra }}-P_{\text {keep }, t}$ offspring, is ranked from top to bottom according to their fitness values as $\left\{\mathbf{q}_{N_{g, p}} ; F_{\text {tra }_{g, p}}\right\}_{p=1}^{P_{\text {tra }}}$.

(4) Mutation: the chromosomes $\mathbf{q}_{N_{g, p}}, 2 \leq p \leq P_{\text {tra }}$, are subject to random mutation with the mutation probability $M_{\text {tra }}$ to explore other area of $\mathscr{A}_{\text {tra }}$ so as to avoid local maxima. Specially, $2 N_{l}$ points of a chromosome, $q_{x, g, p}[n], q_{y, g, p}[n], \cdots, q_{x, g, p}\left[n+N_{l}-1\right], q_{y, g, p}$ $\left[n+N_{l}-1\right]$, are randomly chosen to be mutated. If the mutated chromosome does not satisfy the requirement of $\mathscr{A}_{\text {tra }}$, the chromosome is remutated until the constraints $\mathscr{A}_{\text {tra }}$ are met. Given the mutation probability $M_{\text {tra }}$, a total of $2 M_{\text {tra }} N\left(P_{\text {tra }}-1\right)$ elements will be mutated

The beam sets for the mutated chromosomes are selected with Algorithm 1, and their fitness values are calculated. The mutated population of the $P_{\text {tra }}$ chromosomes is ranked again from top to bottom according to their fitness values as $\left\{\mathbf{q}_{N_{g, p}} ; F_{\text {tra }_{g, p}}\right\}_{p=1}^{P_{\text {tra }}}$.

(5) Termination: if $g>G_{\max , \text { tra }}$, where $G_{\max \text {,tra }}$ denotes the maximum number of generations, the procedure stops with the solution $\mathbf{q}_{N}^{\star}=\mathbf{q}_{N_{g, 1}}$. Otherwise, the procedure repeats from step (2)

This CGA for the UAV-BS trajectory optimization is summarized in Algorithm 3.

3.4. Complexity Analysis. The complexity of the proposed CGA for the UAV-BS deployment optimization with the aid of the greedy SINR beam selection algorithm is specified by the number $N_{\mathrm{GA} \text {,dep }}$ of the cost function evaluations and the complexity per cost function evaluation. Given the population size $P_{\text {dep }}$ and the maximum number of generation $G_{\text {max,dep }}$, we have $N_{\mathrm{GA}}=$ $P_{\text {dep }} \cdot G_{\text {max,dep}}$, whereas the complexity per cost function is derived according to $R_{\text {sum }}$ and the greedy SINR beam selection algorithm. The complexity evaluations of $R_{\text {sum }}$ and the greedy SINR beam selection algorithm are listed in Table 2.

Thus, the complexity of the proposed CGA for the UAV-BS deployment optimization with the aid of the greedy SINR beam selection algorithm is $N_{\mathrm{GA}, \mathrm{dep}} \cdot\{(K-\mid$ $\left.\mathscr{G}_{\mathrm{N}} \mid\right) \sum_{i=1}^{K-\left|\mathscr{G}_{\mathrm{N}}\right|}\left(N_{\mathrm{t}}-\left|\mathscr{G}_{\mathrm{N}}\right|-i\right)\left[3\left(\left|\mathscr{G}_{\mathrm{N}}\right|+i\right)^{3}+4\left(\left|\mathscr{G}_{\mathrm{N}}\right|+i\right)^{2}+1\right]$ $\left.+2 K^{2}+\left(N_{\mathrm{t}}+1\right) K\right\}$. In a similar way, the number $N_{\mathrm{GA} \text {,tra }}$ of the cost function evaluations of the proposed CGA for the UAV-BS trajectory optimization with the aid of the greedy SINR beam selection algorithm is $N_{\mathrm{GA} \text {,tra }}=P_{\text {tra }}$. $G_{\text {max,tra }}$, and the complexity of the proposed CGA for the UAV-BS trajectory optimization with the aid of the greedy SINR beam selection algorithm is $N \cdot N_{\mathrm{GA} \text {,tra }} \cdot\left\{\left(K-\left|\mathscr{G}_{\mathrm{N}}\right|\right)\right.$ $\sum_{i=1}^{K-\left|\mathscr{G}_{\mathrm{N}}\right|}\left(N_{\mathrm{t}}-\left|\mathscr{G}_{\mathrm{N}}\right|-i\right)\left[3\left(\left|\mathscr{G}_{\mathrm{N}}\right|+i\right)^{3}+4\left(\left|\mathscr{G}_{\mathrm{N}}\right|+i\right)^{2}+1\right]+2$ $\left.K^{2}+\left(N_{\mathrm{t}}+1\right) K\right\}$.

\section{Performance Analysis}

We evaluate the performance of the proposed CGA for the UAV-BS deployment optimization and the proposed CGA for the UAV-BS trajectory optimization, with the aid of the greedy SINR beam selection algorithm. We consider an UAV-BS simultaneously serving several $(K)$ ground users in a mmWave system. The terrestrial users are randomly distributed in the square area of $[0,100] \times[0,100]\left(\mathrm{m}^{2}\right)$, and the UAVBS deployment area/flying area is also this $[0,100] \times[0,100]$ $\left(\mathrm{m}^{2}\right)$ square area. The main default parameters of this simulated UAV-BS mmWave system are listed in Table 3.

4.1. Performance of the CGA for UAV-BS Deployment. We first quantify the performance of the proposed CGA-based 


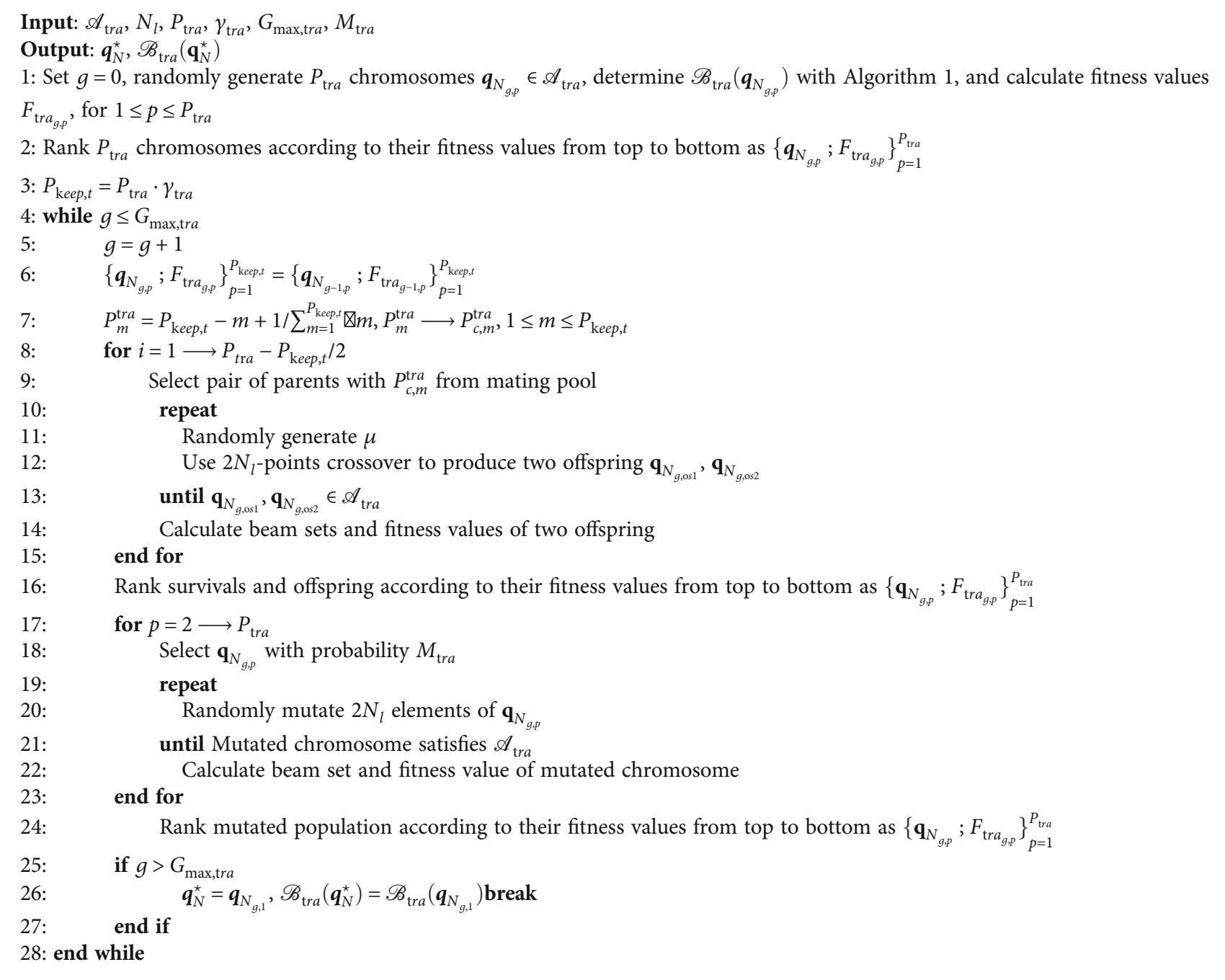

Algorithm 3: Continuous GA for UAV-BS trajectory.

TABle 2: Complexity in number of operations.

\begin{tabular}{lc}
\hline & Number of operations \\
\hline$\left|h_{b, k}^{i}\right|^{2} \forall i, k$ & $N_{\mathrm{t}} K$ \\
$\mathbf{F}$ & $\left(K-\left|\mathscr{G}_{\mathrm{N}}\right|\right) \sum_{i=1}^{K-\left|\mathscr{G}_{\mathrm{N}}\right|}\left(N_{\mathrm{t}}-\left|\mathscr{G}_{\mathrm{N}}\right|-i\right)\left[3\left(\left|\mathscr{G}_{\mathrm{N}}\right|+i\right)^{3}+2\left(\left|\mathscr{G}_{\mathrm{N}}\right|+i\right)^{2}\right]$ \\
$\alpha$ & $\left(K-\left|\mathscr{G}_{\mathrm{N}}\right|\right) \sum_{i=1}^{K-\left|\mathscr{G}_{\mathrm{N}}\right|}\left(N_{\mathrm{t}}-\left|\mathscr{G}_{\mathrm{N}}\right|-i\right)\left(2\left(\left|\mathscr{G}_{\mathrm{N}}\right|+i\right)^{2}+1\right)$ \\
$R_{\text {sum }}$ & $2 K^{2}+K$ \\
\hline
\end{tabular}

UAV-BS deployment with the aid of the greedy SINR beam selection scheme.

Figure 2 depicts the achievable sum rate as the function of the number of users for the proposed CGA-optimized UAV-BS deployment, in comparison with the performance attained by the random UAV-BS deployment. Both deployments are aided by the greedy SINR beam selection. The system has the total transmission power $P_{\mathrm{t}}=20 \mathrm{dBm}$. The position of the mmWave UAV-BS in the random deploy- ment is randomly chosen in the square area of $[0,100] \times$ $[0,100] \mathrm{m}^{2}$. As expected, the CGA-optimized UAV-BS deployment outperforms the random deployment. In particular, for the system with $K=8$ users, the sum rate attained by the proposed optimized approach is about 5 bits/s/Hz higher than that of the random deployment.

We also evaluate the achievable sum rates as the functions of the transmit power $P_{\mathrm{t}}$ for our CGA-optimized UAV-BS deployment and the random UAV-BS deployment 
TABle 3: Default system parameters.

\begin{tabular}{|c|c|c|}
\hline & Parameter & Value \\
\hline \multirow{5}{*}{ Network } & Height of UAV-BS $H$ & $100 \mathrm{~m}$ \\
\hline & Number of transmit antennas at UAV-BS $N_{\mathrm{t}}$ & 16 \\
\hline & AWGN $\sigma_{n}^{2}$ & $-94 \mathrm{dBm}$ \\
\hline & Frequency $f_{m, m}$ & $28 \mathrm{GHz}$ \\
\hline & LoS parameter $\alpha_{\mathrm{L}}$ & 0.95 \\
\hline \multirow{4}{*}{ UAV-BS deployment } & Population size $P_{\text {dep }}$ & 20 \\
\hline & Mutation probability $M_{\mathrm{dep}}$ & 0.2 \\
\hline & Selection ratio $\gamma_{\mathrm{dep}}$ & 0.5 \\
\hline & Number of generation $G_{\text {max,dep }}$ & 50 \\
\hline \multirow{6}{*}{ UAV-BS trajectory } & Population size $P_{\text {tra }}$ & 100 \\
\hline & Mutation probability $M_{\text {tra }}$ & 0.05 \\
\hline & Number of time slots $N$ & 10 \\
\hline & Maximum speed of UAV-BS $v_{\max }$ & $50(\mathrm{~m} / \mathrm{s})$ \\
\hline & Selection ratio $\gamma_{\text {tra }}$ & 0.5 \\
\hline & Number of generation $G_{\mathrm{max} \text {,tra }}$ & 50 \\
\hline
\end{tabular}

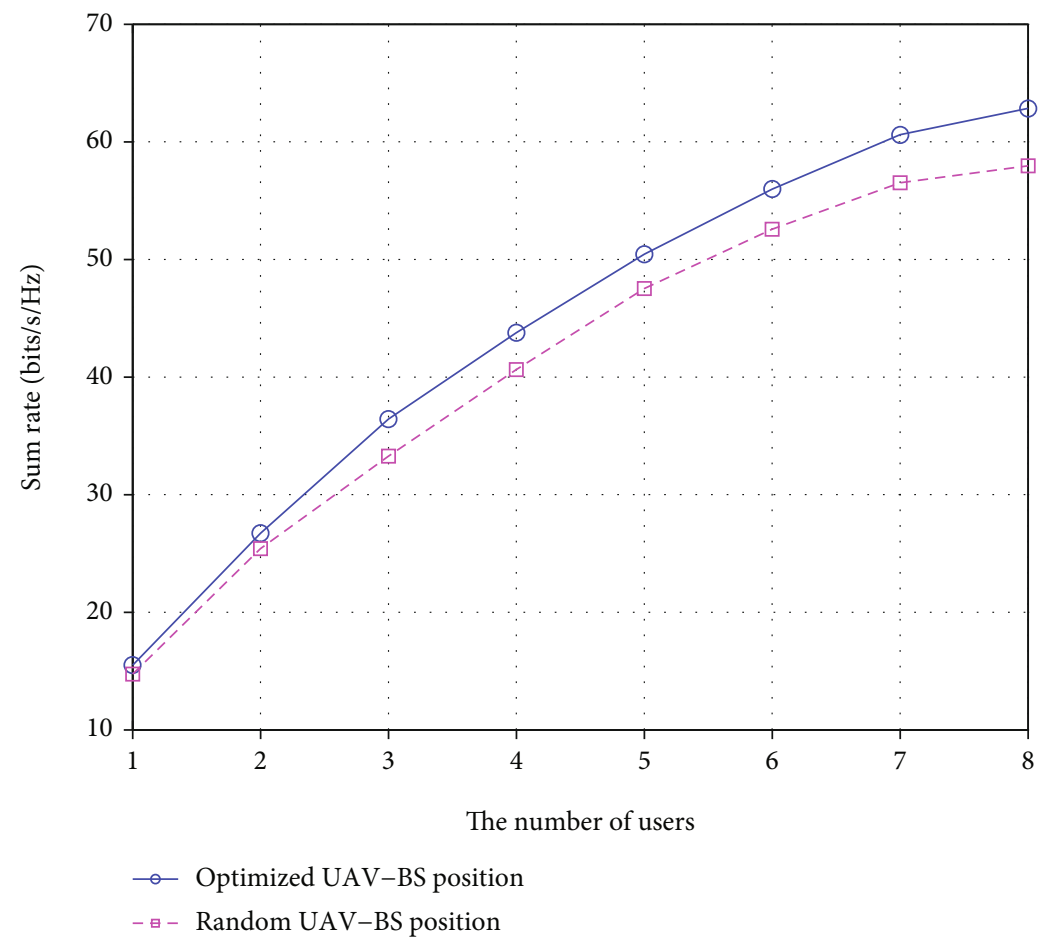

FIGURE 2: Comparison of achievable sum rates versus number of users for the CGA-optimized deployment and random deployment, both with the aid of the greedy SINR beam selection, given total transmission power $P_{\mathrm{t}}=20 \mathrm{dBm}$.

in Figure 3, for the system of $K=4$ ground users. Again, the greedy SINR beam selection is utilized by both deployments. It can be seen that our CGA-optimized UAV-BS deployment outperforms the random deployment by around 8 bits/s/Hz.
Next, we investigate the convergence performance of the proposed CGA with the aid of the greedy SINR beam selection for UAV-BS deployment. The system has $K=4$ ground users with the total transmission power $P_{\mathrm{t}}=20 \mathrm{dBm}$. Figure 4 depicts the maximum sum rate and the mean sum 


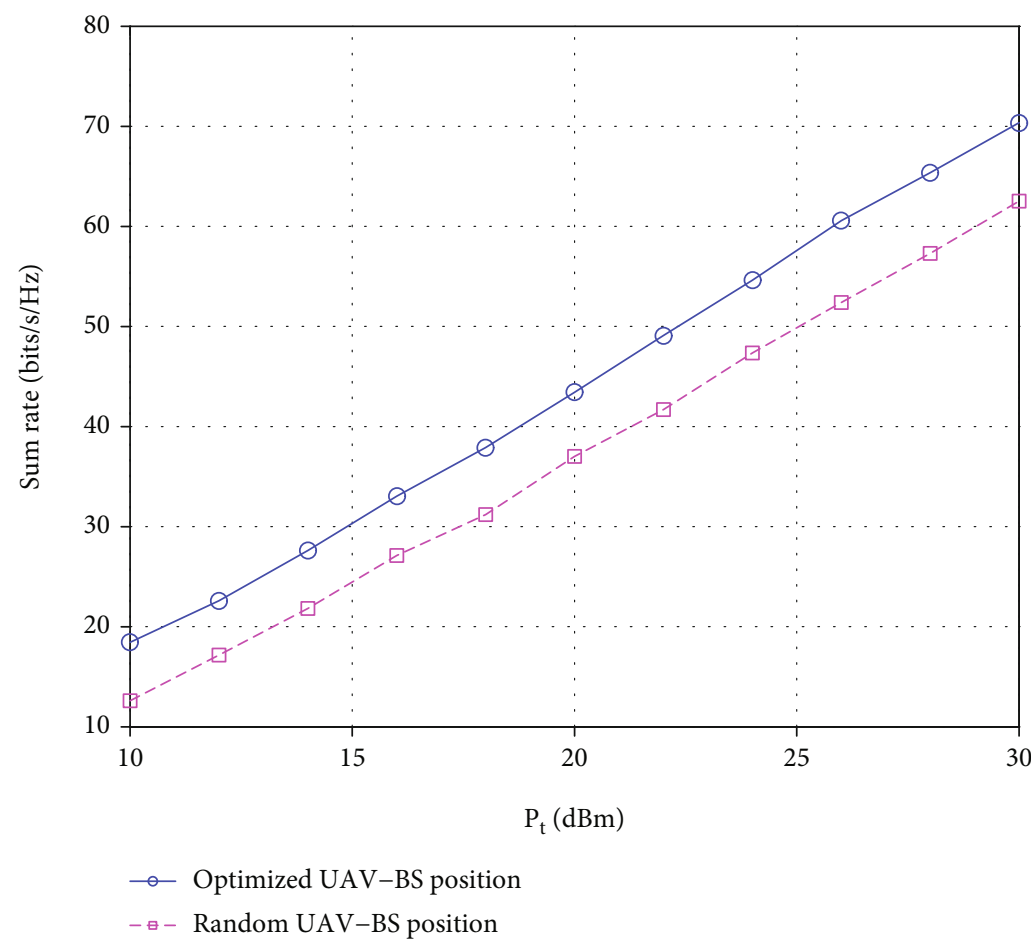

Figure 3: Comparison of achievable sum rates versus total transmit power $P_{\mathrm{t}}$ for the CGA-optimized deployment and random deployment, both with the aid of the greedy SINR beam selection, given $K=4$ ground users.

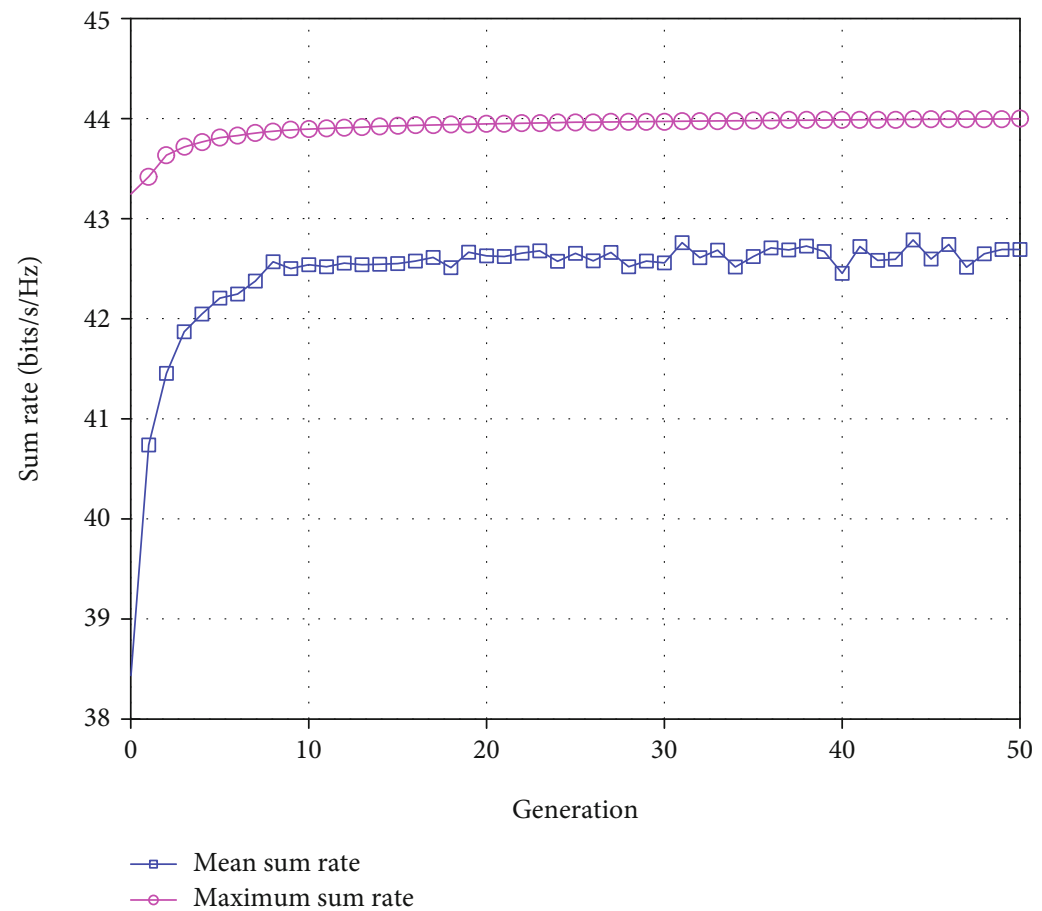

FIGURE 4: Convergence performance of the CGA with the aid of greedy SINR beam selection for optimizing UAV-BS deployment, averaged over 100 runs. The system has $K=4$ ground users and total transmission power $P_{\mathrm{t}}=20 \mathrm{dBm}$. 


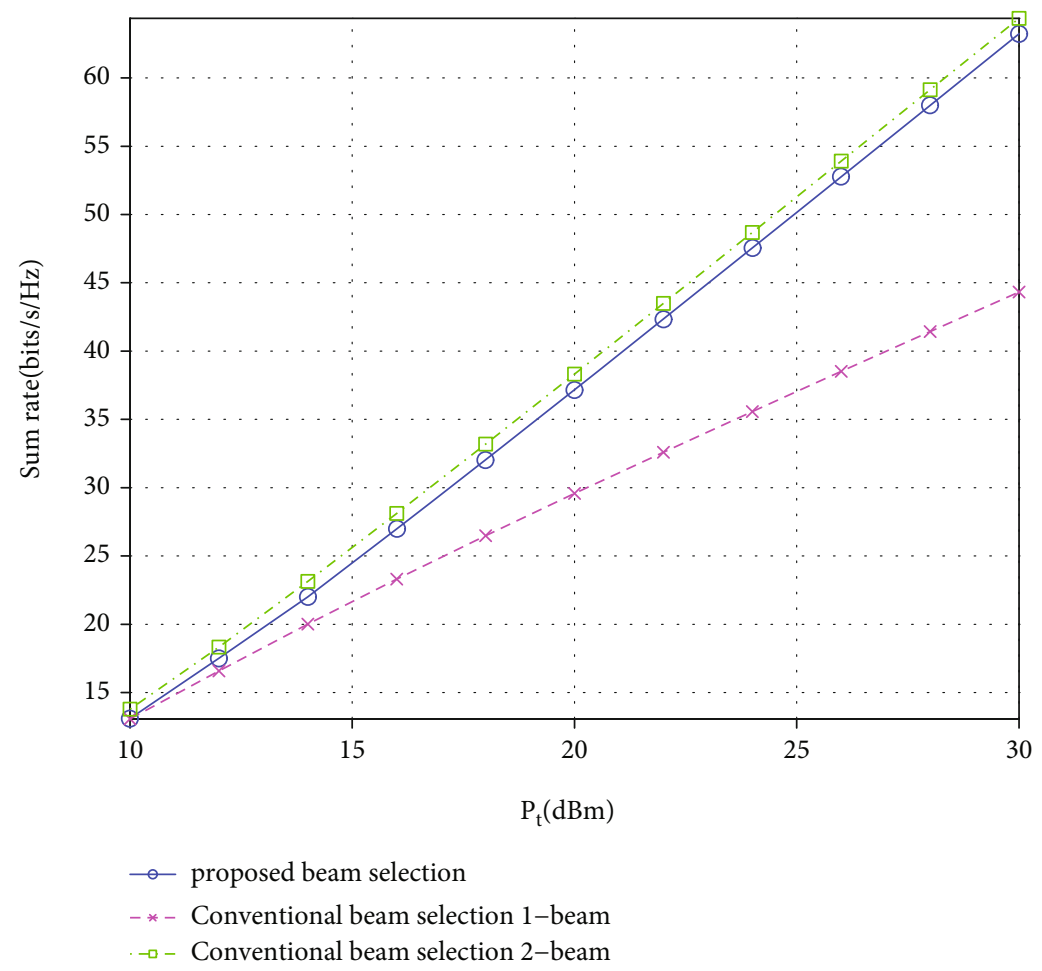

Figure 5: Comparison of achievable sum rates versus total transmit power $P_{\mathrm{t}}$ for three beam selection schemes. The system has $K=4$ ground users, and the UAV-BS is deployed at the CGA-optimized position.

rate of the population as the function of the number of generations, averaged over 100 independent deployments. It can be observed from Figure 4 that the CGA converges within 30 generations.

Our greedy SINR beam selection scheme selects one beam per user by incrementally maximizing the SINR, and it requires $N_{\mathrm{RF}}=K \mathrm{RF}$ chains. The maximum channel magnitude selection (MCMS) scheme in [43] is selected as a reference to the proposed greedy SINR beam selection scheme. For the MCMS scheme, by calculating the $\left|h_{b, k}^{i}\right|$ for each beam, $m$ strongest beams can be chosen for each user. To demonstrate its effectiveness, we compare its sum rate performance versus the system transmit power $P_{\mathrm{t}}$ with those of the MCMS scheme with 1 beam per user and 2 beams per user. Again, the system has $K=4$ ground users. For fair comparison, the CGA-optimized UAV-BS position is used for all the three cases. To mitigate MUI and for a fair comparison, the $\mathrm{ZF}$ precoding is also employed in the MCMS scheme. The MCMS scheme chooses the required number of dominant beams for each user, which may result in selecting the same beam for different users. Note that for the scheme with 2 beams per user, the required RF chains are $N_{\mathrm{RF}}=2 \mathrm{~K}$. Figure 5 compares the sum rate performance of these three beam selection schemes as the functions of the transmission power $P_{\mathrm{t}}$. Observe that our proposed beam selection scheme, which has 1 beam per user, significantly outperforms the MCMS scheme with 1 beam per user. Given $P_{\mathrm{t}}=25 \mathrm{dBm}$, for example, the sum rate of our greedy SINR selection scheme is $13 \mathrm{bits} / \mathrm{s} / \mathrm{Hz}$ higher than that of the latter. This is because this MCMS scheme selects the strongest beam of each user. But some users may share the same strongest beam, and hence, this scheme may result in interference from selecting the same beams for different users. It was further observed that the sum rate of the MCMS scheme with 2 beams per user is only marginally better than that of our greedy SINR selection scheme of 1 beam per user. This is because although utilizing the second strongest beam for each user is beneficial for enhancing the achievable rate of the individual user, some second strongest beams may be shared by different users too, resulting in the increased interference. Hence, the performance gain of the MCMS scheme by using 2 beams per user is very slight compared with our greedy SINR beam selection scheme.

In order to see the trade-off between sum rate performance and RF complexity, we need to investigate the transmit energy efficiency, which is defined by [47]

$$
\eta=\frac{R}{P_{\mathrm{t}}+N_{\mathrm{RF}} \cdot P_{\mathrm{RF}}}(\text { bits } / \mathrm{s} / \mathrm{Hz} / \mathrm{W}),
$$

where $R$ (bits/s/Hz) is the sum rate and $P_{\mathrm{RF}}(\mathrm{W})$ represents the power consumed in each RF chain, and $P_{\mathrm{RF}}=34.4 \mathrm{~mW}$ is adopted as the practical value [47]. For the same system setup as in Figures 5 and 6, compare the energy efficiency against the total transmit power $P_{\mathrm{t}}$ achieved by the three beam selection schemes. As observed from Figure 6, the energy efficiency of the MCMS scheme with 2 beams per user is far inferior to that of its counterpart of 1 beam per 


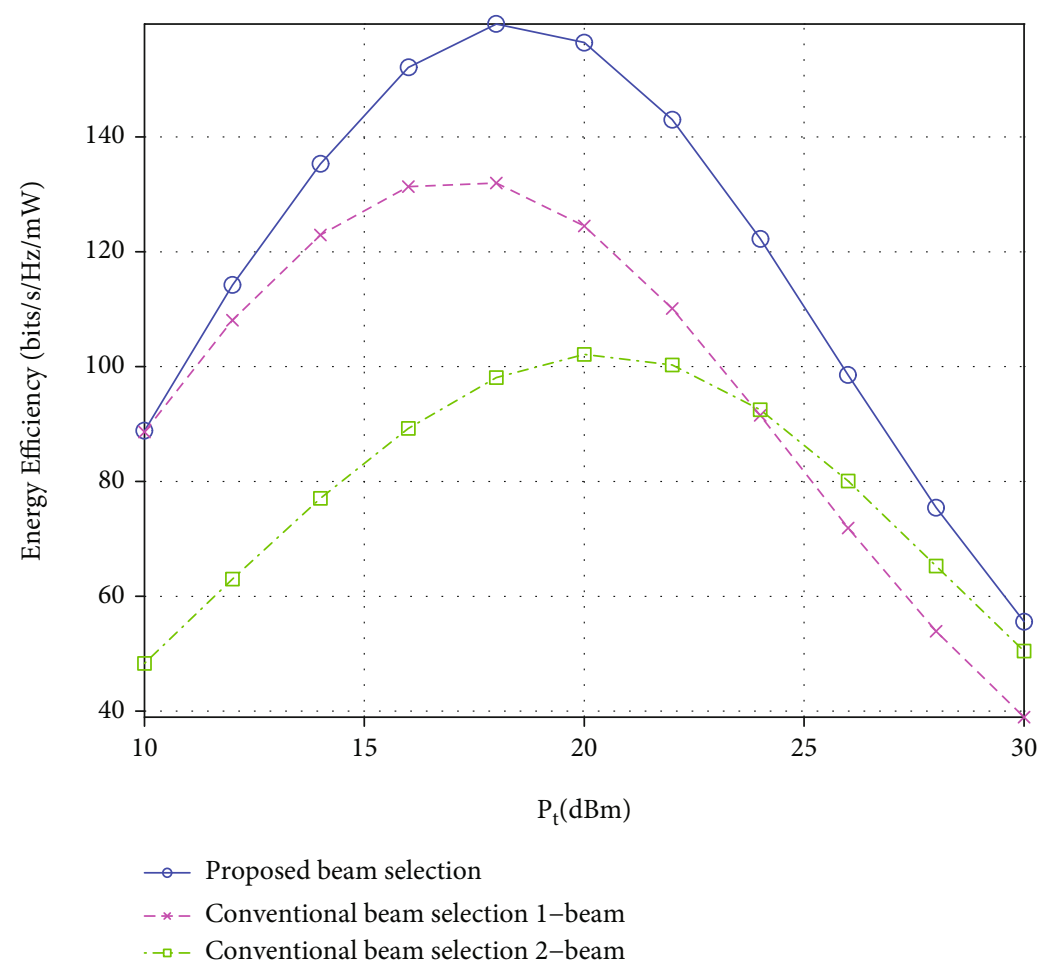

Figure 6: Comparison of energy efficiency performance versus total transmit power $P_{\mathrm{t}}$ for three beam selection schemes. The system has $K=4$ ground users, and the UAV-BS is deployed at the CGA-optimized position.

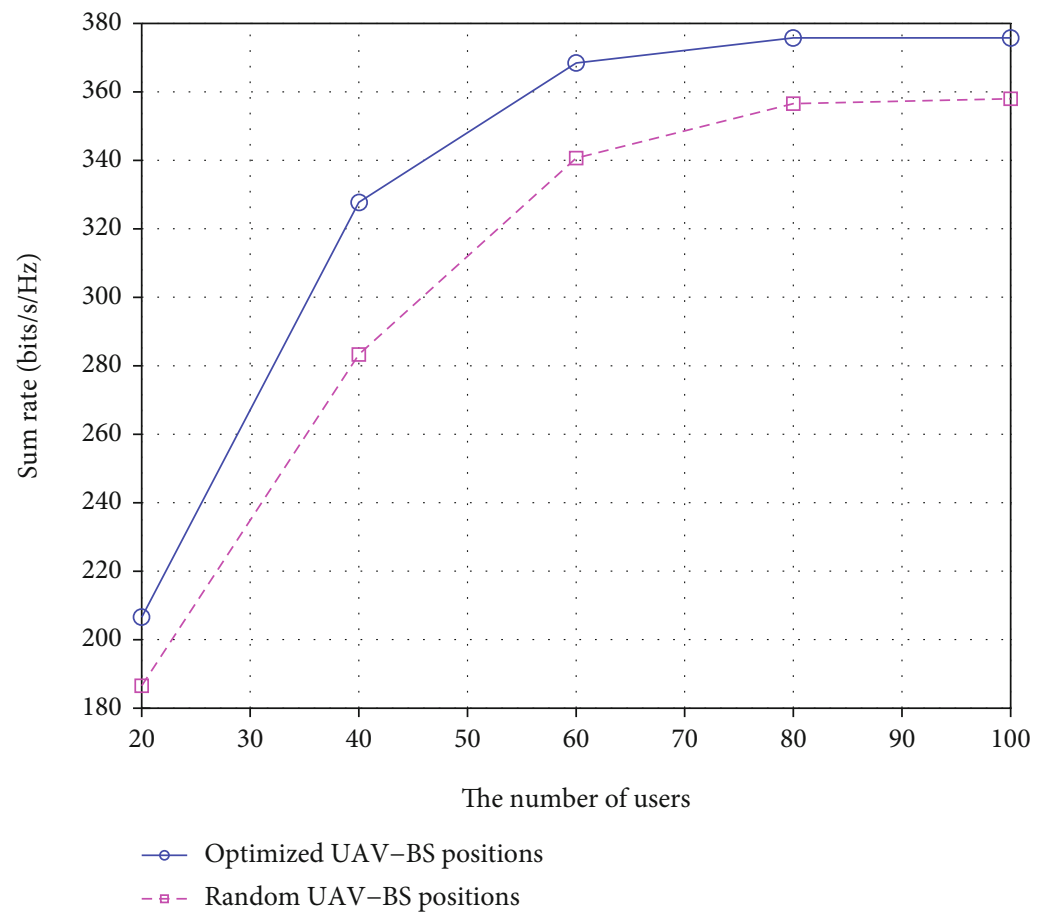

FIGURE 7: Comparison of achievable sum rates versus number of users for the CGA-optimized deployment and random deployment, both with the aid of the greedy SINR beam selection, given total transmission power $P_{\mathrm{t}}=20 \mathrm{dBm}$ and $N_{\mathrm{t}}=256$.

user, except for high $P_{\mathrm{t}}$ situation. Our greedy SINR selection scheme with 1 beam per user significantly outperforms both MCMS schemes, in terms of energy efficiency. In particular, for $P_{\mathrm{t}}=20 \mathrm{~dB}$, although the sum rate of the MCMS scheme with 2 beams per user is about $1 \mathrm{bit} / \mathrm{s} / \mathrm{Hz}$ better than that of our scheme of 1 beam per user, as shown in Figure 5, 


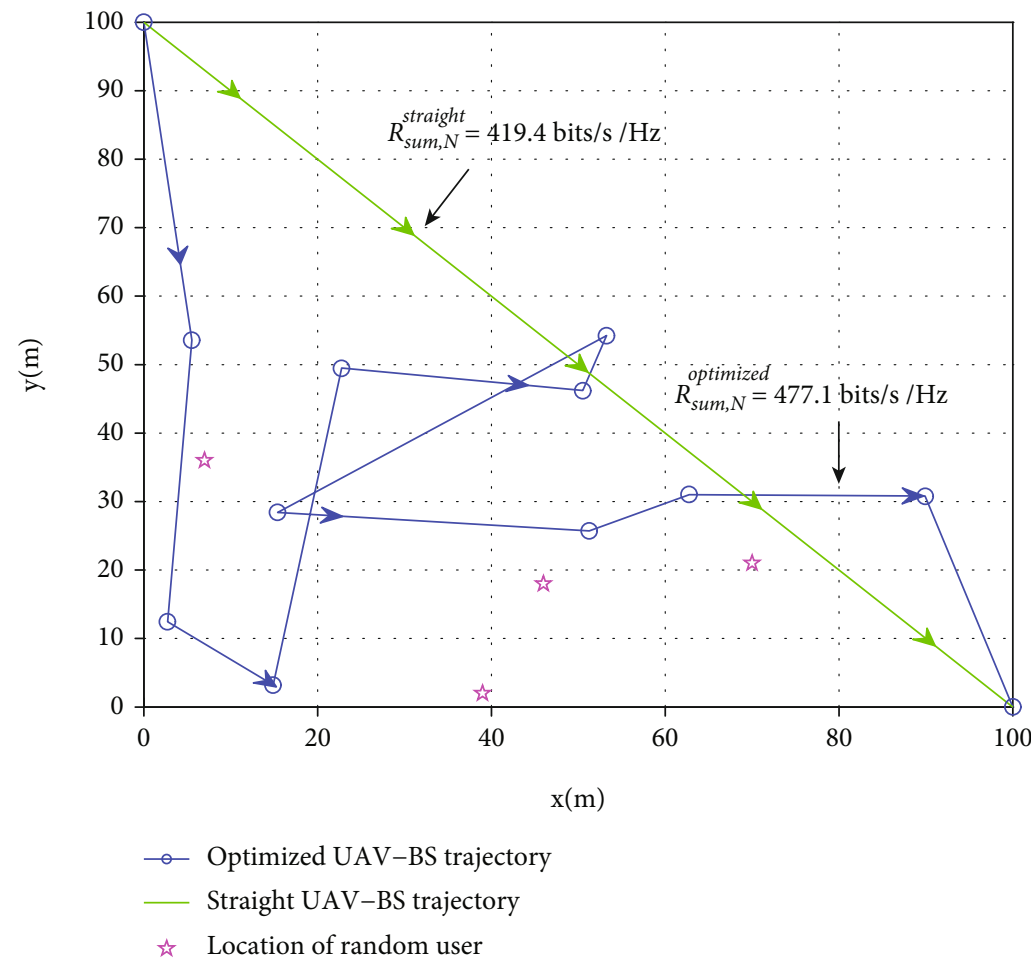

FIGURE 8: Comparison of the CGA-optimized UAV-BS trajectory and the shortest straight-line UAV-BS trajectory, both with the aid of the greedy SINR beam selection scheme. The system has $K=4$ ground users, and the transmit power per time slot is $P_{\mathrm{t} 1}=20 \mathrm{dBm}$.

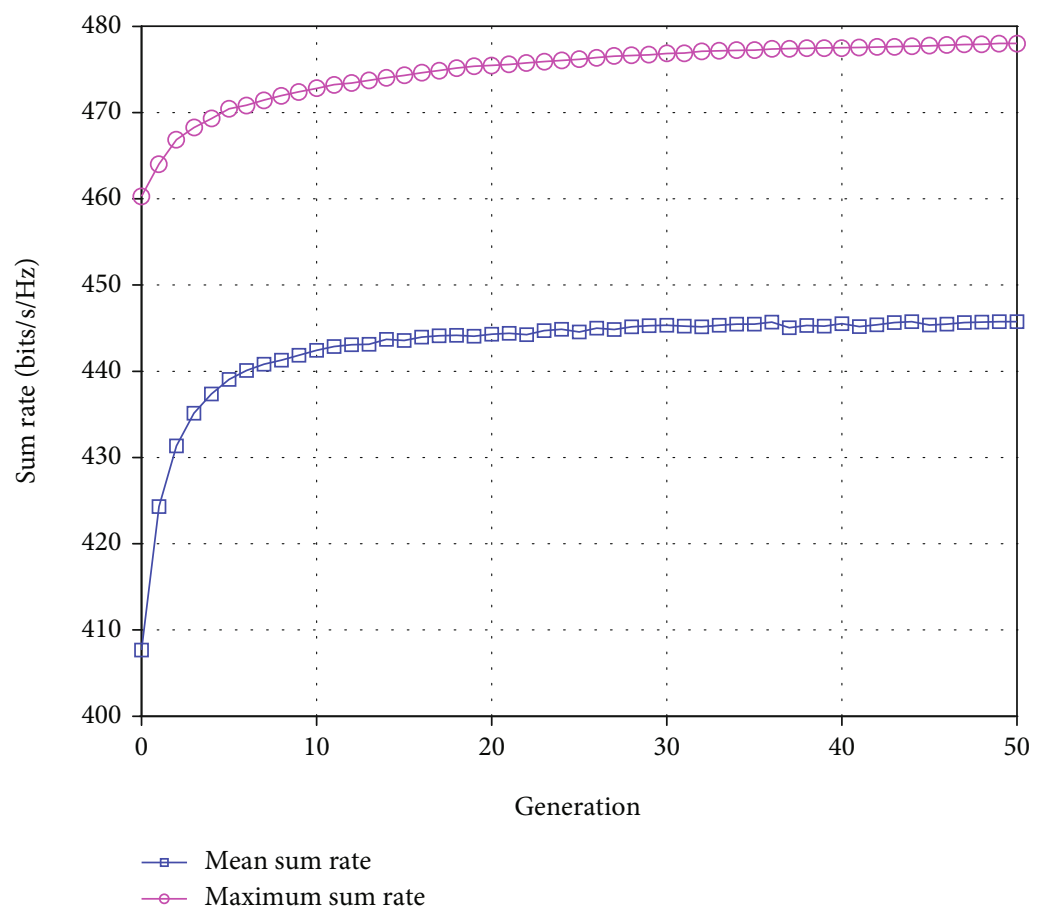

FIGURE 9: Convergence performance of the CGA with the aid of greedy SINR beam selection for optimizing UAV-BS trajectory, averaged over 100 runs. The system has $K=4$ ground users, and transmit power per time slot is $P_{\mathrm{t} 1}=20 \mathrm{dBm}$. 


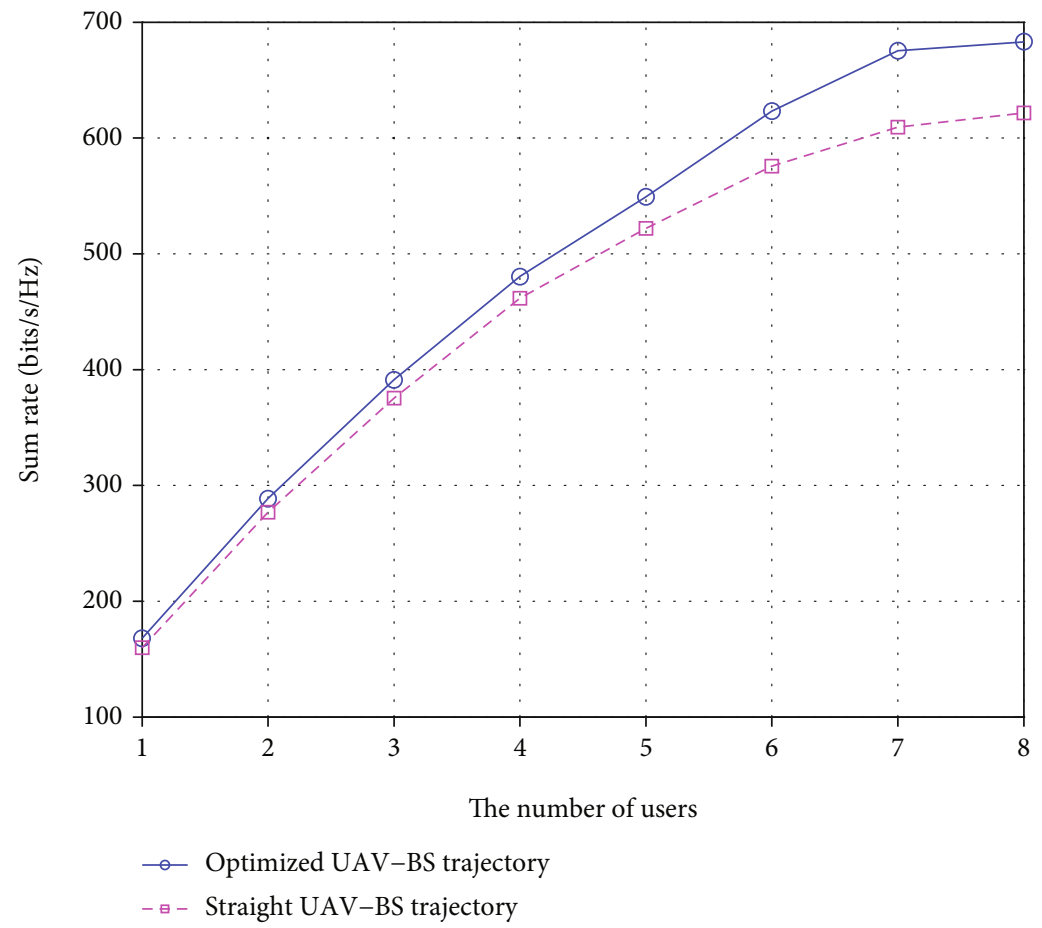

FIGURE 10: Comparison of achievable sum rates versus number of users for the CGA-optimized UAV-BS trajectory and straight-line UAVBS trajectory, both with the aid of greedy SINR beam selection, given transmit power per time slot $P_{\mathrm{t} 1}=20 \mathrm{dBm}$.

the energy efficiency of our greedy SINR beam selection scheme is $57 \mathrm{bits} / \mathrm{s} / \mathrm{Hz} / \mathrm{mW}$ higher than that of the former.

Figure 7 shows the achievable sum rate of the proposed CGA-optimized UAV-BS deployment and the random UAV-BS deployment when $N_{\mathrm{t}}=256$. Both deployments are aided by the greedy SINR beam selection. The system has the total transmission power $P_{\mathrm{t}}=20 \mathrm{dBm}$. As shown in Figure 7 , when the number of users is 100 , the sum rate of the CGA-optimized UAV-BS deployment is better than that of the random deployment. It can conclude that the proposed CGA-optimized UAV-BS deployment is still robust when the number of users is 100 .

4.2. Performance of the CGA for UAV-BS Trajectory. In the first experiment, there are $K=4$ randomly located ground users, whose locations are indicated in Figure 8. The UAVBS flying trajectory starts from $\mathbf{q}_{\mathrm{I}}=[0100]^{T}$ and ends at $\mathbf{q}_{\mathrm{F}}$ $=[1000]^{T}$. The transmit power of the UAV-BS for each time slot is $P_{\mathrm{t} 1}=20 \mathrm{dBm}$. Figure 8 depicts the CGA-optimized UAV-BS trajectory and the straight-line UAV-BS trajectory, both with the aid of the greedy SINR beam selection scheme. For the CGA-optimized UAV-BS trajectory, the UAV-BS is able to serve the ground users better by flying closer to them to achieve a higher sum rate. Specifically, the sum rate of the CGA-optimized UAV-BS trajectory is more than 57 bits/s/Hz higher than that achieved by the straight-line trajectory.

In Figure 9, we investigate the convergence performance of the CGA for optimizing UAV-BS trajectory with the aid of the greedy SINR beam selection scheme for the same system of $K=4$ ground users with the transmit power per time slot $P_{\mathrm{t} 1}=20 \mathrm{dBm}$. The curves of the maximum sum rate and mean sum rate in Figure 9 are averaged over 100 runs. It can be seen that the CGA converges within 50 generations. Evidently, the UAV-BS trajectory optimization is much more challenging than the UAV-BS deployment optimization.

Figure 10 compares the achievable sum rates as the functions of the number of users $K$ for the CGA-optimized UAV-BS trajectory and the straight-line UAV-BS trajectory, both adopting the greedy SINR beam selection. The transmit power per time slot is $P_{\mathrm{t} 1}=20 \mathrm{dBm}$. The UAV-BS's initial and final coordinates are $\mathbf{q}_{\mathrm{I}}=[0100]^{T}$ and $\mathbf{q}_{\mathrm{F}}=[1000]^{T}$, respectively. As shown in Figure 10, the CGA-optimized UAV-BS trajectory outperforms the straight-line UAV-BS trajectory considerably. Furthermore, the sum rate gain of the CGA-optimized UAV-BS trajectory over the straightline UAV-BS trajectory increases with $K$.

Figure 11 compares the achievable sum rates as the functions of transmit power per time slot $P_{\mathrm{t} 1}$ for the CGAoptimized UAV-BS trajectory and the straight-line UAVBS trajectory, both adopting the greedy SINR beam selection. $K=4$ ground users are randomly distributed in the square area of $[0,100] \times[0,100] \mathrm{m}^{2}$. From the results of Figure 11, we observe that the sum rate of the CGAoptimized UAV-BS trajectory is consistently more than 60 bits $/ \mathrm{s} / \mathrm{Hz}$ higher than that achieved by the straight-line UAV-BS trajectory, over the whole range of $P_{\mathrm{t} 1}$ evaluated. When the target sum rate is $600 \mathrm{bits} / \mathrm{s} / \mathrm{Hz}$, the CGAoptimized UAV-BS trajectory attains $2.5 \mathrm{dBm}$ gain in $P_{\mathrm{t} 1}$ compared with the straight-line UAV-BS trajectory. 


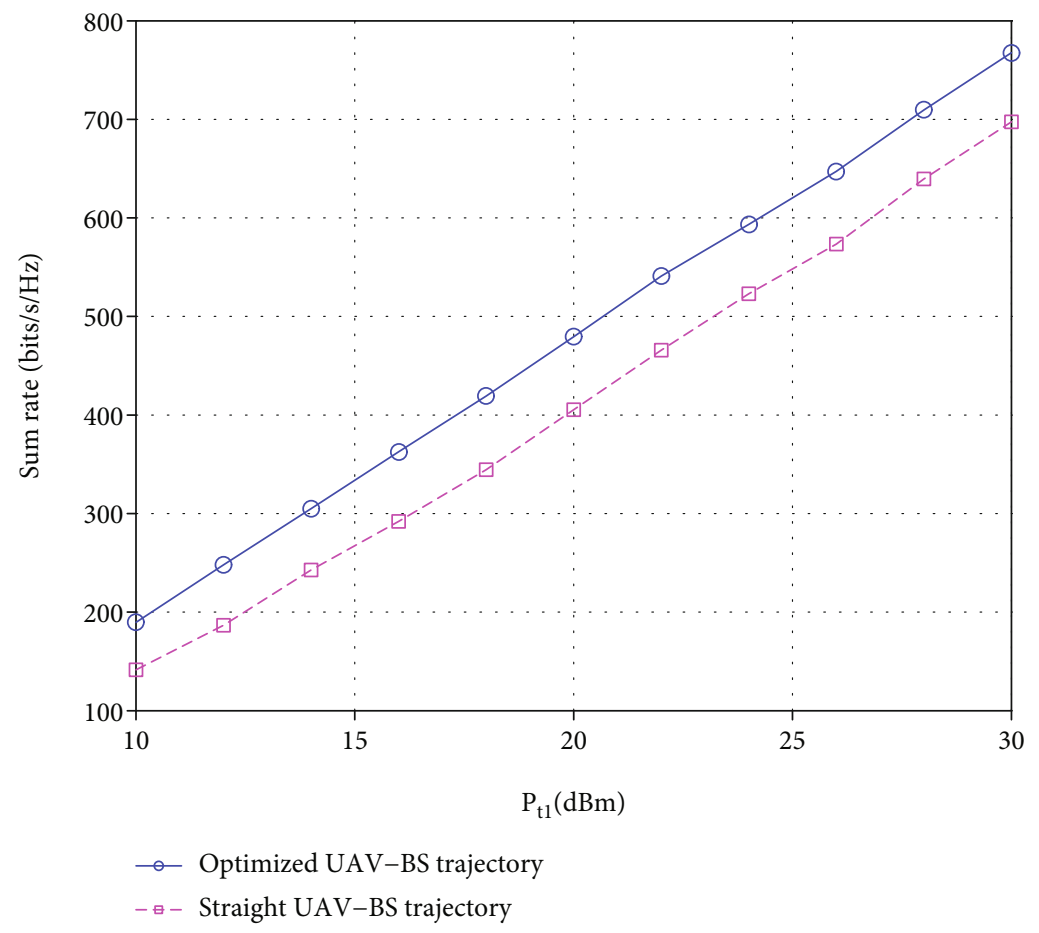

FIGURE 11: Comparison of achievable sum rates versus transmit power per time slot $P_{\mathrm{t} 1}$ for the CGA-optimized UAV-BS trajectory and straight-line UAV-BS trajectory, both adopting greedy SINR beam selection. The system has $K=4$ ground users.

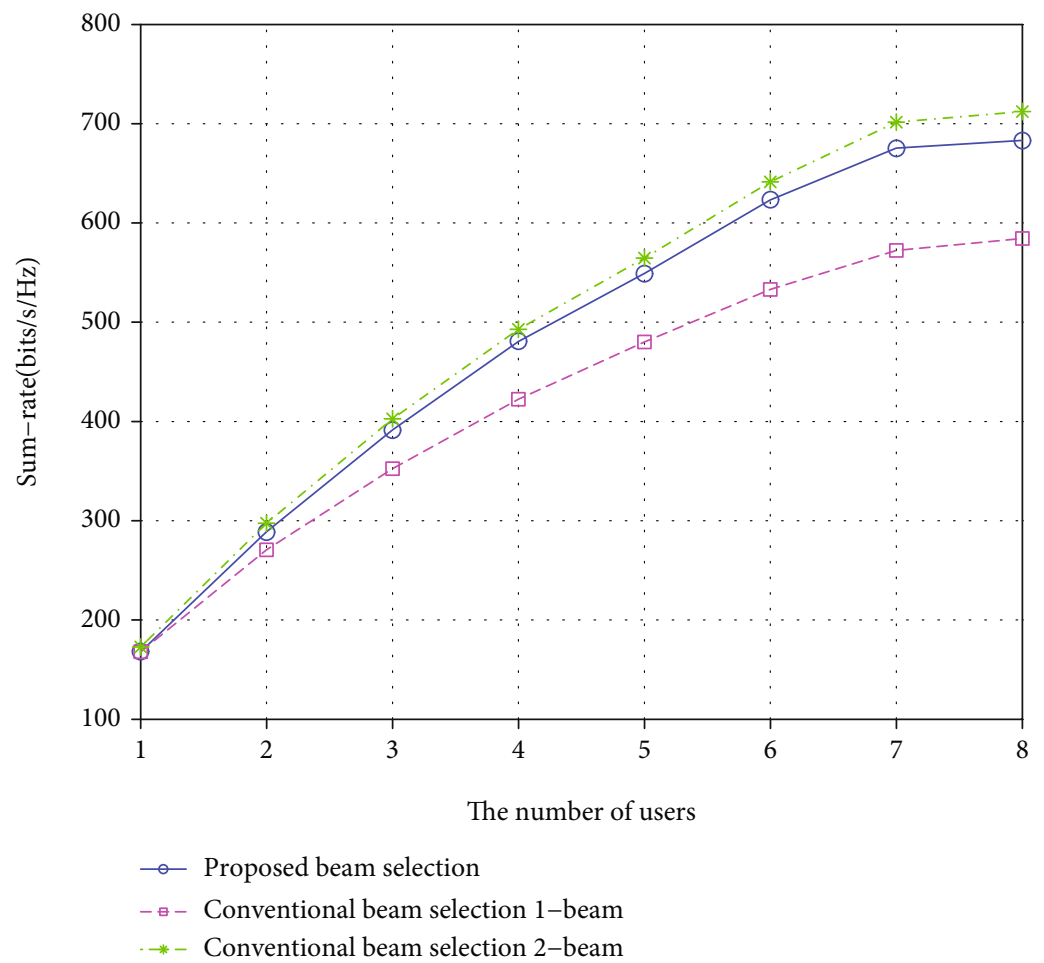

FIGURE 12: Comparison of sum rates versus number of users $K$ for three beam selection schemes, given the system with the CGA-optimized UAV-BS trajectory and transmit power per time slot $P_{\mathrm{t} 1}=20 \mathrm{dBm}$.

For the system adopting the CGA-optimized UAV-BS trajectory with the transmit power per time slot $P_{\mathrm{t} 1}=20$ $\mathrm{dBm}$, Figure 12 compares the sum rates versus the number of ground users for the three beam selection schemes, namely, the proposed greedy SINR beam selection with 1 beam per user as well as the MCMS scheme with 1 beam 


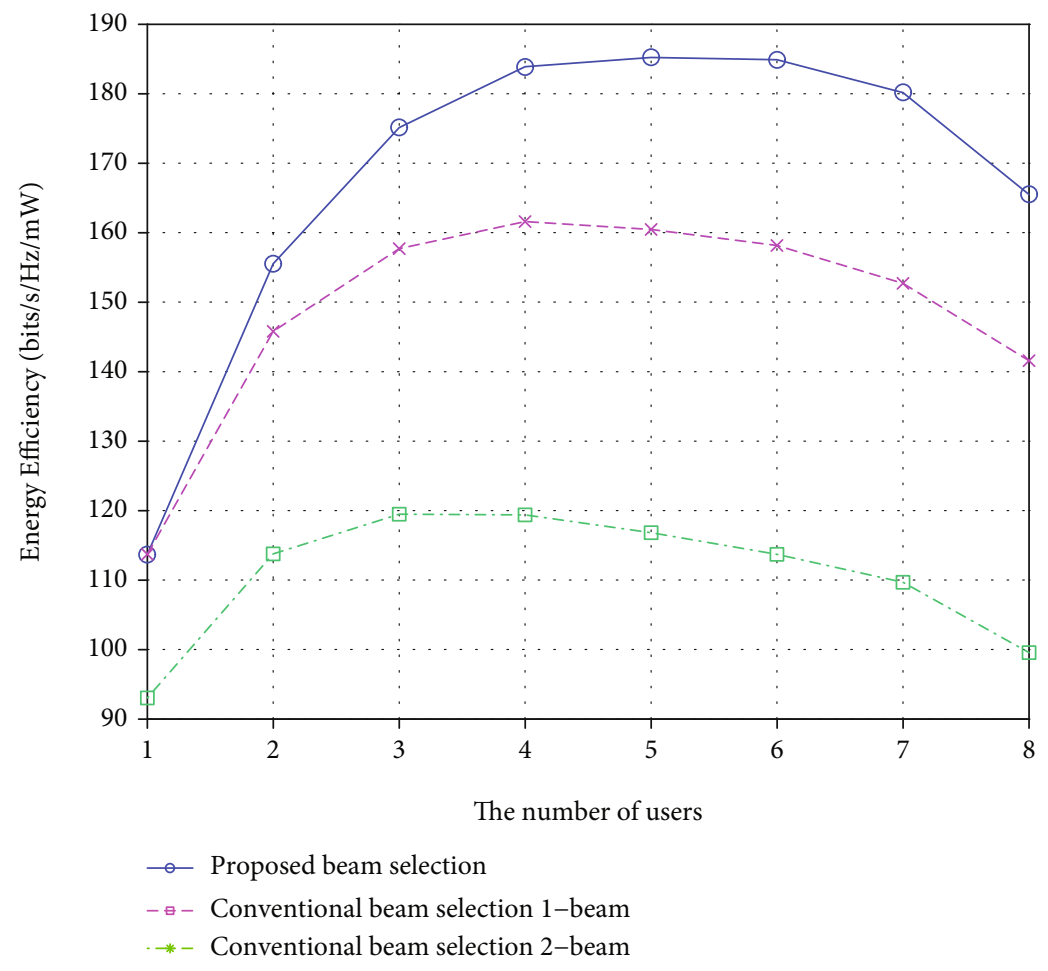

FIGURE 13: Comparison of energy efficiency performance versus number of users for three beam selection schemes for the system with the CGA-optimized UAV-BS trajectory and transmit power per time slot $P_{\mathrm{t} 1}=20 \mathrm{dBm}$.

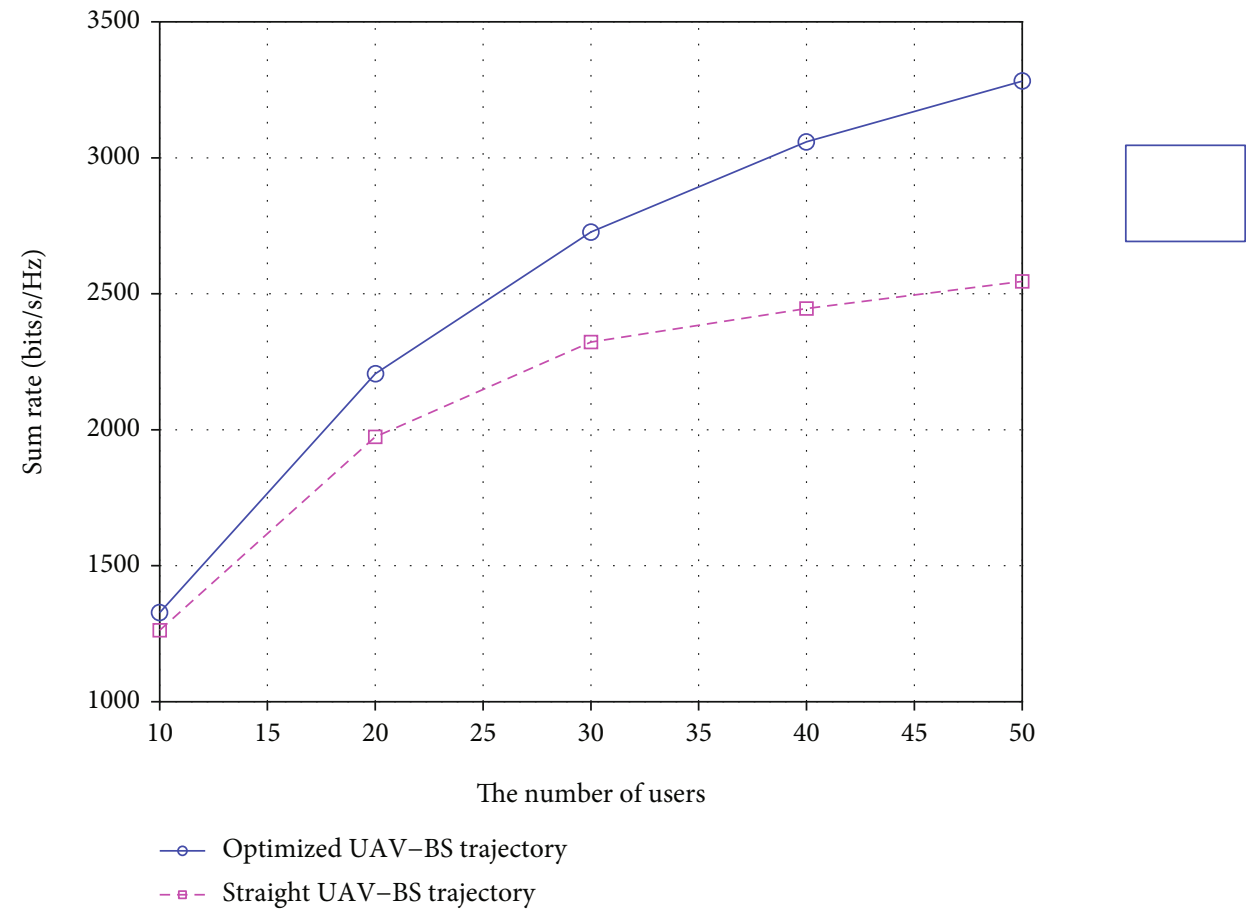

FIGURE 14: Comparison of achievable sum rates versus number of users for the CGA-optimized UAV-BS trajectory and straight-line UAVBS trajectory, both with the aid of greedy SINR beam selection, given transmit power per time slot $P_{\mathrm{t} 1}=20 \mathrm{dBm}$ and $N_{\mathrm{t}}=256$. 
per user and 2 beams per user. It can be seen from Figure 12 that the MCMS scheme with 2 beams per user achieves a slightly better sum rate than our greedy SINR beam selection scheme with 1 beam per user, while our beam selection scheme significantly outperforms the MCMS scheme with 1 beam per user, in terms of sum rate. Our greedy SINR beam selection is the most energy-efficient scheme, while the MCMS scheme with 2 beams per user has the worst energy efficiency, as shown in Figure 13.

Figure 14 compares the achievable sum rates of the CGA-optimized UAV-BS trajectory and the straight-line UAV-BS trajectory with $N_{\mathrm{t}}=256$. Both schemes employ the greedy SINR beam selection. The transmit power per time slot is $P_{\mathrm{t} 1}=20 \mathrm{dBm}$. The UAV-BS's initial and final coordinates are $\mathbf{q}_{\mathrm{I}}=[0100]^{T}$ and $\boldsymbol{q}_{\mathrm{F}}=[1000]^{T}$, respectively. As shown in Figure 10, when the number of users is 50, the sum rate of the CGA-optimized UAV-BS trajectory outperforms that of the straight-line UAV-BS trajectory.

\section{Conclusions}

In this paper, we have investigated the challenging problems of optimizing the UAV-BS deployment and the UAV-BS trajectory in mmWave systems. We have formulated the optimal deployment/trajectory problem as the one that maximizes the sum rate among the ground users subject to the deployment/trajectory constraints. To solve this highly complex and nonconvex problem, we have decomposed it into two subproblems. First, we have proposed a greedy SINR beam selection scheme for the mmWave UAV-BS multiuser system with one beam per user. Specifically, the users with the unique nonsharing dominant beams use their dominant beams, while the other users select the beams by incrementally maximizing the SINR. With the aid of this greedy SINR beam selection, we have proposed to use the CGA to solve the deployment/trajectory optimization problem to maximize the sum rate. The sum rate and energy efficiency as well as convergence performance have been evaluated for the proposed CGA-optimized UAV-BS deployment and CGA-optimized UAV-BS trajectory. Our studies have demonstrated that the proposed solutions achieve excellent performance, in terms of both sum rate and energy efficiency. The results have also shown that the CGA converges sufficiently fast even for the very challenging UAV-BS trajectory optimization.

Our future work will consider that each user is equipped with multiple antennas. The receive beamforming and beam selection can be designed to compensate for the path loss in mmWave frequency.

\section{Data Availability}

The simulation data used to support the findings of this study are included within the article. The MATLAB code data used to support the findings of this study are available from the corresponding author upon request.

\section{Conflicts of Interest}

The authors declare that they have no conflicts of interest.

\section{References}

[1] Z. Xiao, P. Xia, and X.-G. Xia, "Enabling UAV cellular with millimeter-wave communication: potentials and approaches," IEEE Communications Magazine, vol. 54, no. 5, pp. 66-73, 2016.

[2] J. Zhang, T. Chen, S. Zhong et al., "Aeronautical ad hoc networking for the internet-above-the-clouds," Proceedings of the IEEE, vol. 107, no. 5, pp. 868-911, 2019.

[3] Y. Zeng, R. Zhang, and T. J. Lim, "Wireless communications with unmanned aerial vehicles: opportunities and challenges," IEEE Communications Magazine, vol. 54, no. 5, pp. 36-42, 2016.

[4] X. Li, Q. Wang, Y. Liu, T. A. Tsiftsis, Z. Ding, and A. Nallanathan, "UAV-aided multi-way NOMA networks with residual hardware impairments," IEEE Wireless Communications Letters, vol. 9, no. 9, pp. 1538-1542, 2020.

[5] C. Pan, H. Ren, Y. Deng, M. Elkashlan, and A. Nallanathan, "Joint blocklength and location optimization for URLLCenabled UAV relay systems," IEEE Communications Letters, vol. 23, no. 3, pp. 498-501, 2019.

[6] Z. Yang, C. Pan, K. Wang, and M. Shikh-Bahaei, "Energy efficient resource allocation in UAV-enabled mobile edge computing networks," IEEE Transactions on Wireless Communications, vol. 18, no. 9, pp. 4576-4589, 2019.

[7] M. M. Azari, F. Rosas, K.-C. Chen, and S. Pollin, "Optimal $\mathrm{UAV}$ positioning for terrestrial-aerial communication in presence of fading," in Proc. GLOBECOM 2016, pp. 1-7, Washington, DC, USA, 2016.

[8] X. Xi, X. Cao, P. Yang, J. Chen, T. Quek, and D. Wu, "Joint user association and UAV location optimization for UAVaided communications," IEEE Wireless Communications Letters, vol. 8, no. 6, pp. 1688-1691, 2019.

[9] M. Alzenad, A. El-Keyi, F. Lagum, and H. Yanikomeroglu, “3$\mathrm{D}$ placement of an unmanned aerial vehicle base station (UAV-BS) for energy-efficient maximal coverage," IEEE Wireless Communications Letters, vol. 6, no. 4, pp. 434-437, 2017.

[10] Q. Wu, Y. Zeng, and R. Zhang, "Joint trajectory and communication design for multi-UAV enabled wireless networks," IEEE Transactions on Wireless Communications, vol. 17, no. 3, pp. 2109-2121, 2018.

[11] Y. Cai, F. Cui, Q. Shi, M. Zhao, and G. Y. Li, "Dual-UAVenabled secure communications: joint trajectory design and user scheduling," IEEE Journal on Selected Areas in Communications, vol. 36, no. 9, pp. 1972-1985, 2018.

[12] N. Zhao, X. Pang, Z. Li et al., "Joint trajectory and precoding optimization for UAV-assisted NOMA networks," IEEE Transactions on Communications, vol. 67, no. 5, pp. 3723 3735, 2019.

[13] Y. Zeng and R. Zhang, "Energy-efficient UAV communication with trajectory optimization," IEEE Transactions on Wireless Communications, vol. 16, no. 6, pp. 3747-3760, 2017.

[14] M. R. Akdeniz, Y. Liu, M. K. Samimi et al., "Millimeter wave channel modeling and cellular capacity evaluation," IEEE Journal on Selected Areas in Communications, vol. 32, no. 6, pp. 1164-1179, 2014.

[15] S. Rangan, T. S. Rappaport, and E. Erkip, "Millimeter-wave cellular wireless networks: potentials and challenges," Proceedings of the IEEE, vol. 102, no. 3, pp. 366-385, 2014.

[16] C. Zhang, W. Zhang, W. Wang, L. Yang, and W. Zhang, "Research challenges and opportunities of UAV millimeter- 
wave communications," IEEE Wireless Communications, vol. 26, no. 1, pp. 58-62, 2019.

[17] M. Mozaffari, W. Saad, M. Bennis, Y. H. Nam, and M. Debbah, "A tutorial on UAVs for wireless networks: applications, challenges, and open problems," IEEE Communications Surveys and Tutorials, vol. 21, no. 3, pp. 2334-2360, 2019.

[18] L. Zhu, J. Zhang, Z. Xiao, X. Cao, D. O. Wu, and X. G. Xia, “3$\mathrm{D}$ beamforming for flexible coverage in millimeter-wave UAV communications," IEEE Wireless Communications Letters, vol. 8, no. 3, pp. 837-840, 2019.

[19] N. Tafintsev, M. Gerasimenko, D. Moltchanov et al., "Improved network coverage with adaptive navigation of mmWave-based drone-cells," in Proc. GLOBECOM 2018 Workshops, pp. 1-7, Abu Dhabi, United Arab Emirates, 2018.

[20] M. Gapeyenko, I. Bor-Yaliniz, S. Andreev, H. Yanikomeroglu, and Y. Koucheryavy, "Effects of blockage in deploying mmWave drone base stations for $5 \mathrm{G}$ networks and beyond," in Proc. ICC 2018 Workshops, pp. 1-6, Kansas City, MO, USA, 2018.

[21] Z. Xiao, H. Dong, L. Bai, D. O. Wu, and X. G. Xia, "Unmanned aerial vehicle base station (UAV-BS) deployment with millimeter-wave beamforming," IEEE Internet of Things Journal, vol. 7, no. 2, pp. 1336-1349, 2020.

[22] X. Li, M. Zhao, M. Zeng et al., "Hardware impaired ambient backscatter NOMA systems: reliability and security," IEEE Transactions on Communications, vol. 69, no. 4, pp. 27232736, 2021.

[23] R. Jia, X. Chen, C. Zhong, D. W. Ng, H. Lin, and Z. Zhang, "Design of non-orthogonal beamspace multiple access for cellular Internet-of-things," IEEE Journal of Selected Topics in Signal Processing, vol. 13, no. 3, pp. 538-552, 2019.

[24] J. Brady, N. Behdad, and A. M. Sayeed, "Beamspace MIMO for millimeter-wave communications: system architecture, modeling, analysis, and measurements," Institute of Electrical and Electronics Engineers, vol. 61, no. 7, pp. 3814-3827, 2013.

[25] X. Gao, L. Dai, Z. Chen, Z. Wang, and Z. Zhang, "Near-optimal beam selection for beamspace mmWave massive MIMO systems," IEEE Communications Letters, vol. 20, no. 5, pp. 1054-1057, 2016.

[26] P. V. Amadori and C. Masouros, "Low RF-complexity millimeter-wave beamspace-MIMO systems by beam selection," IEEE Transactions on Communications, vol. 63, no. 6, pp. 2212-2223, 2015.

[27] C. Sun, X. Gao, and Z. Ding, "BDMA in multicell massive MIMO communications: power allocation algorithms," IEEE Transactions on Signal Processing, vol. 65, no. 11, pp. 29622974, 2017.

[28] Z. Jiang, S. Zhou, and Z. Niu, "Optimal discrete spatial compression for beamspace massive MIMO signals," IEEE Transactions on Signal Processing, vol. 66, no. 9, pp. 2480-2493, 2018.

[29] S. Chen, Y. Wu, and S. McLaughlin, "Genetic algorithm optimization for blind channel identification with higher order cumulant fitting," IEEE Transactions on Evolutionary Computation, vol. 1, no. 4, pp. 259-265, 1997.

[30] K. Yen and L. Hanzo, "Genetic algorithm assisted joint multiuser symbol detection and fading channel estimation for synchronous CDMA systems," IEEE Journal on Selected Areas in Communications, vol. 19, no. 6, pp. 985-998, 2001.

[31] J. Zhang, S. Chen, X. Mu, and L. Hanzo, "Evolutionary-algorithm-assisted joint channel estimation and turbo multiuser detection/decoding for OFDM/SDMA," IEEE Transactions on Vehicular Technology, vol. 63, no. 3, pp. 1204-1222, 2014.

[32] R. L. Haupt and S. E. Haupt, Practical Genetic Algorithms, John Wiley \& Sons, New Jersey, USA, 2nd edition, 2004.

[33] F. Al-Turjman, J. P. Lemayian, S. Alturjman, and L. Mostarda, "Enhanced deployment strategy for the 5G drone-BS using artificial intelligence," IEEE Access, vol. 7, pp. 75999-76008, 2019.

[34] H. Ren, C. Pan, K. Wang, Y. Deng, M. Elkashlan, and A. Nallanathan, "Achievable data rate for URLLC-enabled UAV systems with 3-D channel model," IEEE Wireless Communications Letters, vol. 8, no. 6, pp. 1587-1590, 2019.

[35] Z. Xiao, L. Zhu, J. Choi, P. Xia, and X. G. Xia, "Joint power allocation and beamforming for non-orthogonal multiple access (NOMA) in 5G millimeter wave communications," IEEE Transactions on Wireless Communications, vol. 17, no. 5, pp. 2961-2974, 2018.

[36] A. Alkhateeb, O. El Ayach, G. Leus, and R. W. Heath, "Channel estimation and hybrid precoding for millimeter wave cellular systems," IEEE Journal of Selected Topics in Signal Processing, vol. 8, no. 5, pp. 831-846, 2014.

[37] T. S. Rappaport, Y. Xing, M. C. GR, A. F. Molisch, E. Mellios, and J. Zhang, "Overview of millimeter wave communications for fifth-generation (5G) wireless networks-with a focus on propagation models," Institute of Electrical and Electronics Engineers, vol. 65, no. 12, pp. 6213-6230, 2017.

[38] C. Yan, L. Fu, J. Zhang, and J. Wang, "A comprehensive survey on UAV communication channel modeling," IEEE Access, vol. 7, pp. 107769-107792, 2019.

[39] Y. Zeng, J. Xu, and R. Zhang, "Energy minimization for wireless communication with rotary-wing UAV," IEEE Transactions on Wireless Communications, vol. 18, no. 4, pp. 23292345, 2019.

[40] D. Tse and P. Viswanath, Fundamentals of Wireless Communication, Cambridge University Press, Cambridge, U.K., 2005.

[41] A. M. Sayeed and N. Behdad, "Continuous aperture phased MIMO: a new architecture for optimum line-of-sight links," in Proc. APSURSI 2011, pp. 293-296, Spokane, WA, USA, 2011.

[42] A. M. Sayeed, "Deconstructing multiantenna fading channels," IEEE Transactions on Signal Processing, vol. 50, no. 10, pp. 2563-2579, 2002.

[43] A. Sayeed and J. Brady, "Beamspace MIMO for highdimensional multiuser communication at millimeter-wave frequencies," in Proc. GLOBECOM 2013, pp. 3679-3684, Atlanta, GA, USA, 2013.

[44] C. Masouros, M. Sellathurai, and T. Ratnarajah, "Large-scale MIMO transmitters in fixed physical spaces: the effect of transmit correlation and mutual coupling," IEEE Transactions on Communications, vol. 61, no. 7, pp. 2794-2804, 2013.

[45] M. Mitchell, An Introduction to Genetic Algorithms, MIT press, Cambridge, MA, 1998.

[46] L. Hanzo, L.-L. Yang, E. Kuan, and K. Yen, Single- and MultiCarrier DS-CDMA: Multi-User Detection, Space-Time Spreading, Synchronisation, Networking and Standards, John Wiley \& Sons, New Jersey, USA, 2003.

[47] C. Masouros, M. Sellathurai, and T. Ratnarajah, "Computationally efficient vector perturbation precoding using thresholded optimization," IEEE Transactions on Communications, vol. 61, no. 5, pp. 1880-1890, 2013. 